\title{
High-Performance Distributed Brillouin Optical Fiber Sensing
}

\author{
Yongkang DONG* \\ National Key Laboratory of Science and Technology on Tunable Laser, Harbin Institute of Technology, Harbin 150001, \\ China \\ *Corresponding author: Yongkang DONGＥ-mail: aldendong@163.com
}

\begin{abstract}
This paper reviews the recent advances on the high-performance distributed Brillouin optical fiber sensing, which include the conventional distributed Brillouin optical fiber sensing based on backward stimulated Brillouin scattering and two other novel distributed sensing mechanisms based on Brillouin dynamic grating and forward stimulated Brillouin scattering, respectively. As for the conventional distributed Brillouin optical fiber sensing, the spatial resolution has been improved from meter to centimeter in the time-domain scheme and to millimeter in the correlation-domain scheme, respectively; the measurement time has been reduced from minute to millisecond and even to microsecond; the sensing range has reached more than $100 \mathrm{~km}$. Brillouin dynamic grating can be used to measure the birefringence of a polarization-maintaining fiber, which has been explored to realize distributed measurement of temperature, strain, salinity, static pressure, and transverse pressure. More recently, forward stimulated Brillouin scattering has gained considerable interest because of its capacity to detect mechanical features of materials surrounding the optical fiber, and remarkable works using ingenious schemes have managed to realize distributed measurement, which opens a brand-new way to achieve position-resolved substance identification.
\end{abstract}

Keywords: Distributed sensing; stimulated Brillouin scattering; Brillouin dynamic grating; multi-parameter measurement

Citation: Yongkang DONG, "High-Performance Distributed Brillouin Optical Fiber Sensing," Photonic Sensors, 2021, 11(1): 6990.

\section{Introduction}

The distributed Brillouin optical fiber sensors have gained significant attention in the past few decades, due to the feasibility of strain and temperature measurements and their applications in the monitoring of infrastructures [1, 2]. The conventional distributed Brillouin optical fiber sensing is based on the backward stimulated Brillouin scattering, where the strain or temperature is a function of the Brillouin frequency shift (BFS), and the distributed strain or temperature can be demodulated from the Brillouin gain spectra (BGS) over the sensing fiber. Over the past two decades, many efforts have been made to improve its performances, including spatial resolution, measurement time, and sensing range.

The spatial resolution is a key property to distinguish the small-size events with distinct temperature/strain state. Brillouin optical timedomain analysis (BOTDA) [3] and Brillouin optical correlation-domain analysis (BOCDA) [4] are usually used to obtain a high spatial resolution. In the BOTDA scheme, the pump wave is pulse

Received: 19 November 2020 / Revised: 15 December 2020

(C) The Author(s) 2021. This article is published with open access at Springerlink.com

DOI: $10.1007 / \mathrm{s} 13320-021-0616-7$

Article type: Review 
modulated for the distributed sensing, and the spatial resolution is determined by the pulse width. The acoustic wave lifetime of $10 \mathrm{~ns}$ has imposed the pulse width limit for the associated spatial resolution to be $1 \mathrm{~m} \mathrm{[5].} \mathrm{Several} \mathrm{techniques} \mathrm{have} \mathrm{been}$ proposed to realize a sub-meter spatial resolution in the time-domain scheme, including the dark-pulse technique [6], pulse pre-pump (PPP) technique [7], differential pulse-width (DPP) technique [8-10], and Brillouin echo technique [11]. In the BOCDA scheme, the stimulated Brillouin scattering (SBS) is localized at a specific position within the sensing fiber, due to the correlation between two modulated counter-propagating optical waves. There are several specific modulation methods to create the correlation peak, which are the frequency modulation [4], phase modulation [12, 13], amplified spontaneous emission (ASE) [14], and chaotic laser [15]. The principle of the BOCDA allows it to circumvent the pulse-based spatial resolution limit, so that the spatial resolution can be obtained at the order of centimeter and even millimeter [16].

The acquiring of the BGS over the sensing fiber usually involves a laser frequency scanning, which is a time-consuming process leading to a long measurement time [17]. Generally, the measurement time for a conventional Brillouin optical fiber sensing ranges from a few seconds to tens of minutes, which limits its applications to a static or a slowly-varying measurement. To reduce the measurement time, several novel fast schemes are proposed, and their common point is to avoid frequency scanning or speed up the frequency switching. These schemes include: optical frequency comb technique [18-22], slope-assisted technique [23-29], optical frequency-agile technique [30-33], and the optical chirp chain (OCC) technique [34-38], based on which the measurement time has been reduced to millisecond and even to microsecond.

Extending the sensing range for distributed Brillouin optical fiber sensors is always a perpetual topic, and a long sensing distance is vital for the monitoring of large-scale infrastructures, such as oil/gas pipelines and power lines [39-58]. However, due to the Kerr effect $[39,40]$ and non-local effect [41-43], the sensing range is usually limited to a few tens of kilometers. To extend the sensing range, several techniques have been proposed, such as Raman amplification [44-46], time division multiplexing [47], frequency division multiplexing [48], pulse coding [49-51], wide-bandwidth frequency modulation [52], and image processing methods [53], based on which the sensing range has reached more than $100 \mathrm{~km}$.

For the conventional distributed Brillouin optical fiber sensing based on backward stimulated Brillouin scattering, the measurable parameters are limited to temperature and strain. Recently, two novel distributed sensing mechanisms based on Brillouin dynamic grating (BDG) and forward stimulated Brillouin scattering (FSBS) have been explored to realize multi-parameter sensing. The BDG has been widely studied since it was proposed for the first time to achieve optical storage in 2007 [59]. In general, when two beams of pump light (their frequency difference equals to BFS of the optical fiber) with the same polarization state are injected into the fiber, the coherent acoustic field can be excited by the SBS effect forming the BDG, which has been widely used in characterization of optical fibers [60-65], optical storage [59, 66], all-optical signal processing [67-69], microwave photonics [70-72], high-precision spectral analysis [73], and optical fiber sensing [74-82]. Using a polarization-maintaining fiber, the $\mathrm{BDG}$ has been explored to realize distributed measurement of temperature, strain, salinity, static pressure, and transverse pressure.

FSBS has been used in a wide variety of photonic applications, such as high-performance lasers [83, 84], optical isolators and circulators [85], frequency comb generation [86], and metrology [87-89]. Recently, its capability has been extended 
to chemical sensing through measuring of acoustic impedance of materials surrounding the optical fiber [90, 91]. However, the forward scattering characteristic renders FSBS sensor incapable of spatially-resolved distributed measurements. More recently, several remarkable works based on opto-mechanical time-domain reflectometry (OMTDR) [92], local light phase recovery (LPR) [93], and opto-mechanical time-domain analysis (OMTDA) [94] have been independently proposed to realize distributed FSBS measurement.

This paper reviews the recent advances on the high-performance conventional distributed Brillouin optical fiber sensing based on backward stimulated Brillouin scattering and two other novel distributed sensing based on the BDG and FSBS, respectively.

\section{Distributed sensing based on backward stimulated Brillouin scattering}

\subsection{Principle}

When a high power optical wave propagates along an optical fiber, a moving density grating (i.e., the acoustic wave) can be excited in the core of the optical fiber via an electrostriction effect through the SBS effect, which will induce a Doppler shift (i.e., the BFS) for its backscattered Stokes wave and the $\mathrm{BFS}$ is given by [2]

$$
v_{B}=\frac{\Omega_{B}}{2 \pi} \equiv \frac{2 n_{\text {eff }} V_{A}}{\lambda_{p}}
$$

where $n_{\text {eff }}$ is the effective core refractive index, $\Omega_{B}$ is the angular frequency of the BFS, $V_{A}$ is the acoustic velocity, and $\lambda_{p}$ is the wavelength of the pump wave in vacuum. Generally, for a silica single-mode fiber (SMF), the BFS is $10 \mathrm{GHz}-$ $11 \mathrm{GHz}$ at the communication wavelength of $1550 \mathrm{~nm}$. When the counter-propagating probe wave is around the BFS shifted from the pump wave, it will strengthen the acoustic wave by means of electrostriction. With the grown intensity of the acoustic wave, more pump wave is scattered, and then the intensity of the probe wave is increased, so that the acoustic and probe waves mutually reinforce the intensity of each other. The above SBS process can be expressed by the coupled three-wave equations [2]:

$$
\begin{gathered}
\frac{\partial E_{p}}{\partial z}+\frac{n}{c} \frac{\partial E_{p}}{\partial t}=\mathrm{i} \kappa E_{s} \rho \\
-\frac{\partial E_{s}}{\partial z}+\frac{n}{c} \frac{\partial E_{s}}{\partial t}=\mathrm{i} \kappa E_{p} \rho^{*} \\
\frac{\partial \rho}{\partial t}+\left(\frac{1}{2} \Gamma_{B}+\mathrm{i}\left(\Omega_{B}-\Omega\right)\right) \rho=\mathrm{i} \Lambda E_{p} E_{s}^{*}
\end{gathered}
$$

where $E_{p}, E_{s}$, and $\rho$ are defined to be the field amplitudes of the pump wave, the probe wave, and the acoustic wave, respectively; $n$ is the effective index; $c$ is the velocity in vacuum; $\Omega$ is the angular frequency offset between the pump and probe waves; $\Gamma_{B}$ is the linewidth of the BGS; $\kappa$ and $\Lambda$ denote the elasto-optic and electrostrictive coupling coefficients, respectively.

It is noted that the BFS has a good linear relationship with the strain and temperature of the fiber and is given by the equation below:

$$
v_{B}=C_{B}^{\varepsilon} \Delta \varepsilon+C_{B}^{T} \Delta T+v_{B 0}
$$

where $v_{B 0}$ is the initial BFS of the fiber, $C_{B}^{\varepsilon}$ and $C_{B}^{T}$ are the strain and temperature coefficients of the BFS, respectively, and $\Delta \mathcal{E}$ and $\Delta T$ are the variations of strain and temperature. Based on (3), the distributed variations of the strain or temperature can be demodulated by measuring the distributed BFS, as shown in Fig. 1.

\subsection{High-spatial-resolution distributed sensing}

For the conventional BOTDA sensors, it is unpractical to obtain a sub-meter spatial resolution with an optical pulse of less than $10 \mathrm{~ns}$. The acoustic wave lifetime of $10 \mathrm{~ns}$ has imposed the pulse width limit for the associated spatial resolution to be $1 \mathrm{~m}$ [5]. The impacts of a short pulse ( $<10 \mathrm{~ns})$ degrade the measurement accuracy due to the broadened Brillouin spectrum and drastically reduce Brillouin interaction [5]. Based on the pre-excitation of the acoustic wave, several impressive techniques have been developed, including the dark-pulse technique, 
pulse pre-pump (PPP) technique, differential pulse-width pair (DPP) technique, and Brillouin echo technique. In Fig. 2, the detailed pulse modulation on the pump wave is shown for each technique. In the dark-pulse and PPP schemes, the spatial resolution is determined by the width of the short pulse $(\Delta t)$. In the other two schemes, a high spatial resolution is enabled by using two pump pulses with a slight difference $(\Delta t)$ on the width or phase [90].

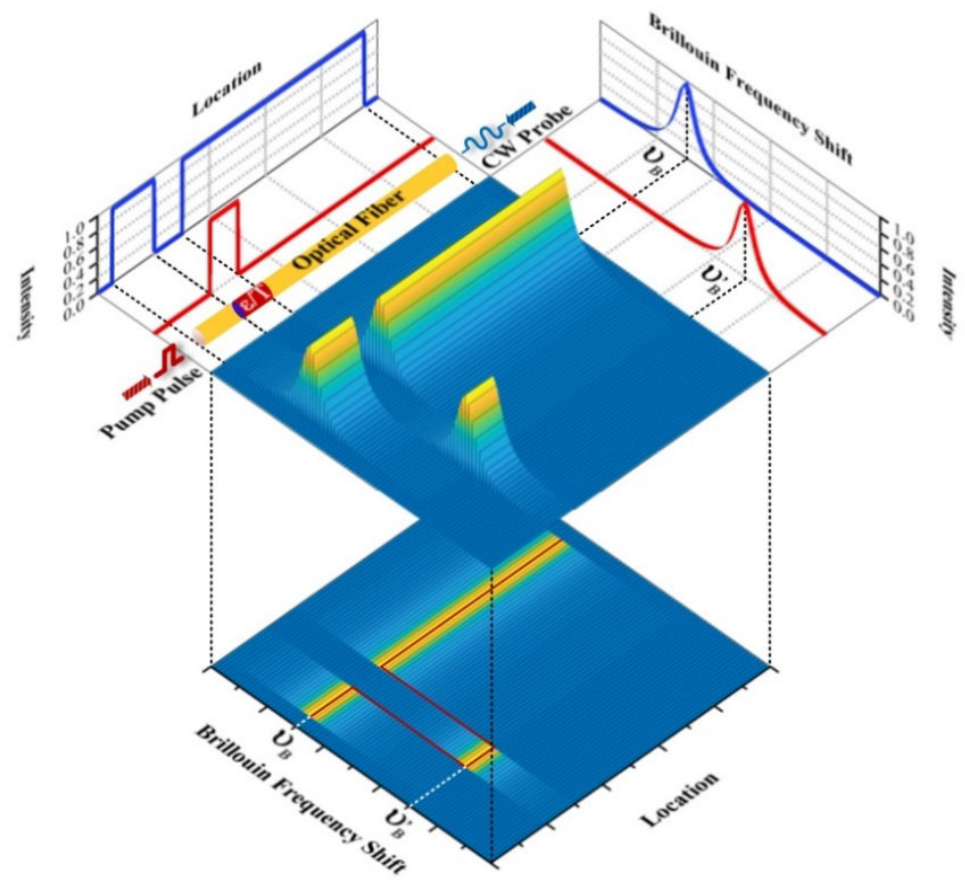

Fig. 1 Principle of distributed sensing based on backward stimulated Brillouin scattering.

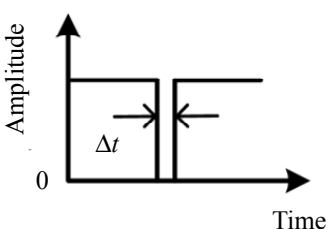

(a)

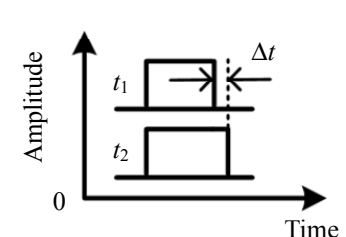

(c)

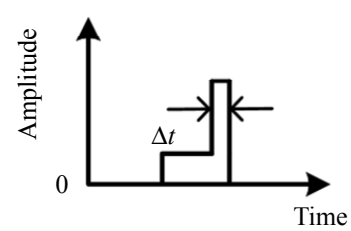

(b)

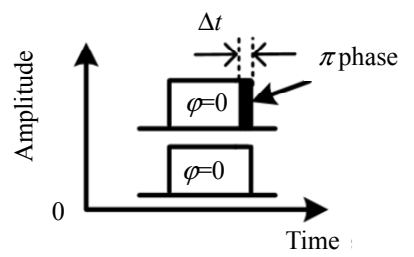

(d)
Fig. 2 Four main techniques and the corresponding pulse modulation methods: (a) dark-pulse technique, (b) PPP technique, (c) DPP te chnique, and (d) Brillouin echo technique [95].

The dark-pulse technique has been proposed by Brown et al. [6] in 2005, which is able to achieve a high spatial resolution and overcome the above two obstacles (i.e., broadened spectrum and reduced intensity). The system is working in the Brillouin loss scheme. The anti-Stokes wave is a continuous wave $(\mathrm{CW})$, while the pump wave is dark pulse modulated as a quasi-CW as shown in Fig. 2(a). The linewidth of the measured BGS is equal to its natural value, which is able to maintain a high measurement accuracy. As a result, it is able to realize a $5-\mathrm{cm}$ spatial resolution over a $100-\mathrm{m}$ sensing fiber [6], which further is optimized to 2-cm spatial resolution with a 200-ps dark pulse [96]. Due to the non-local response of the sensor, it is hard to distinguish a small BFS variation in a small-size hotspot. As a result, the dark-pulse based Brillouin sensing can only be used for a high-spatial-resolution measurement when the BFS difference between the hotspot and rest fiber is bigger than the linewidth of the intrinsic BGS. Moreover, the sensing length is also limited to kilometer scale, in the case of the onset of the SBS [91].

The presentence of a low power DC leakage 
light is also able to pre-excite the acoustic wave before the arrival of the bright pulse [5]. However, the measured BGS of a short fiber section is distorted if the strain or temperature is small. To reduce the pulse base contribution on the BGS and maintain the useful component, a modified PPP-BOTDA has been proposed [7], and the schematic diagram is shown in Fig. 2(b). A spatial resolution of $10 \mathrm{~cm}$ is achieved with a $13 \mathrm{~ns}$ pre-pump pulse and a $1 \mathrm{~ns}$ pump pulse [97]. Besides, the pump depletion is mitigated and the Brillouin threshold is increased, so that the sensing range can be extended. However, the width of the pre-pump pulse should also be optimized to introduce less non-local information at an acceptable signal-to-noise ratio (SNR). As a result, the PPP-BOTDA is able to realize a sub-meter spatial resolution with a narrow Brillouin spectrum, but a system measurement error is also introduced by the non-local response of the sensor.

In the DPP-BOTDA scheme, two long duration pulses are used for two separate measurements and the differential Brillouin signal is obtained by making subtraction between the two pulse-width signals [8]. The width of the differential BGS is determined by the long duration pulses, which can provide a good frequency resolution. The spatial resolution is determined by the difference between two pulse widths, and meanwhile, the minimum effective spatial resolution is limited by the falling time of the optical pulse. Using the $8 / 8.2 \mathrm{~ns}$ pulse-width pair with a $150 \mathrm{ps}$ falling time, it is able to achieve a $2 \mathrm{~cm}$ spatial resolution over a $2 \mathrm{~km}$ sensing fiber [9], as showing in Fig. 3. In 2008, the differential pulse-phase pair technique was proposed based on the Brillouin echo technique [98]. In the other measurement, the pump wave is only pulse modulated with no phase modulation, as the other pulse in Fig. 2(d). The DPP and Brillouin echo techniques utilize the differential measurements with two pump pulses, and these two types of sensors are free from non-local response. Besides, the intensity of the Brillouin echo differential signal is twice as much as that of the DPP one, when using the same experimental parameters. Therefore, the Brillouin echo owns a better SNR and a measurement accuracy, which manifests itself as a promising tool to achieve a high spatial resolution in a mid-to-long distance. Meanwhile, the DPP-BOTDA has also been widely used in the laboratory investigations and practical applications, as the implementation is simple and easy.

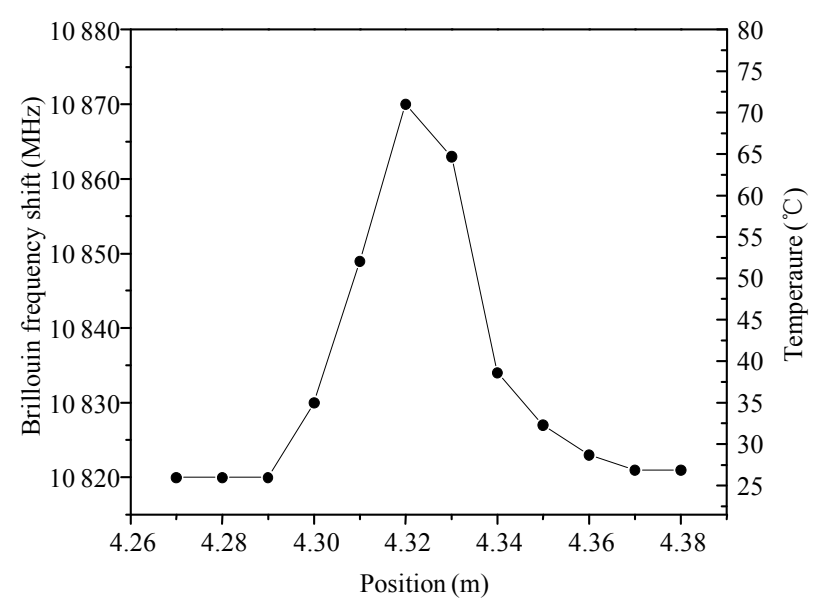

Fig. 3 Measured BFS and corresponding temperature as a function of the position. A $2-\mathrm{cm}$ segment located at $4.31 \mathrm{~cm}-$ $4.33 \mathrm{~cm}$ is heated to $73{ }^{\circ} \mathrm{C}$ [9].

In order to circumvent the spatial resolution limit of the pulse-based scheme, a novel technique has been proposed by using the correlation between the pump and probe waves. The SBS interaction is generated at the position with a high correlation (i.e., correlation peak), while it is suppressed outside the correlation peak. The correlation peak can be obtained by several specific modulation methods, which are the frequency modulation [4], phase modulation [12, 13], amplified spontaneous emission [14], and chaotic laser [15]. Among these techniques, the frequency modulation and phase modulation schemes are widely used, as shown in Fig. 4. In 1999, the correlation-based technique was proposed to interrogate the Brillouin gain spectrum, which is enabled by the frequency modulated pump and probe waves [99]. In order to obtain a high correlation, the pump and probe waves are 
sinusoidal frequency modulated at the frequency $f_{m}$ with a modulation amplitude $\Delta f$. The pump and probe waves are counter-propagating, and the frequency difference between these two waves is only fixed at the correlation peaks. In this case, the Brillouin interaction is constant and steady, so that there is no broadening effect on the BGS. Meanwhile, it is able to map the BGS by scanning the frequency offset between the pump and probe waves. At other positions, the frequency of the pump wave changes continuously relative to the probe wave, resulting in a suppression of the Brillouin interaction, as shown in Fig. 4(a). To obtain an ultra-high spatial resolution $(1.6 \mathrm{~mm})$ [16], the modulation frequency $f_{m}$ is varying from $19.4 \mathrm{MHz}$ to $19.5 \mathrm{MHz}$ depending on the measurement position, while the amount of $\Delta f$ is increased to $33 \mathrm{GHz}$.

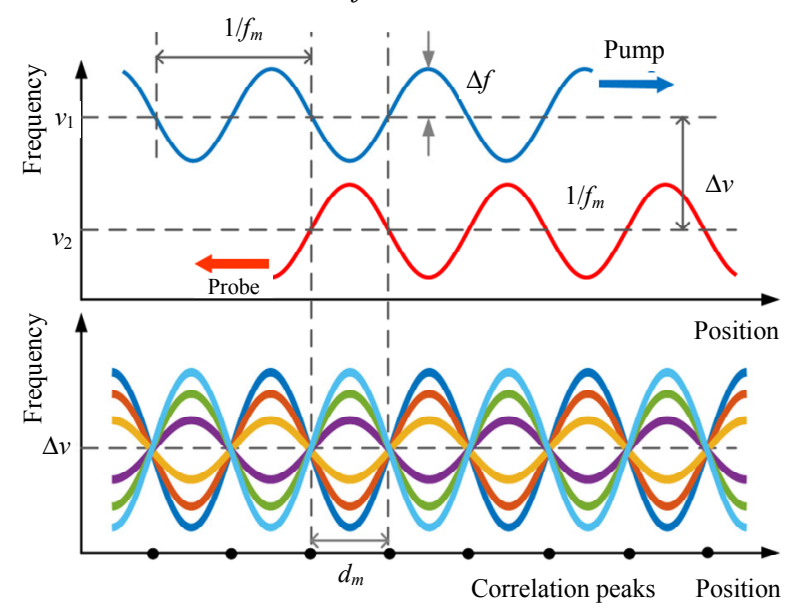

(a)

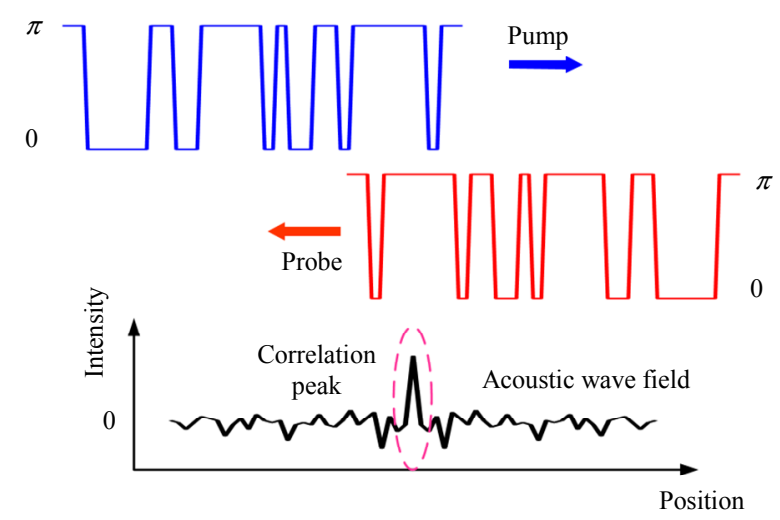

(b)

Fig. 4 Operation principle of BOCDA: (a) frequency modulation and (b) phase modulation [95].
In 2012, another type of the CW-based BOCDA was realized with the optical phase modulation technique [12]. The operation principle of the phase modulation based BOCDA is illustrated in Fig.4(b), where the pump and probe waves are simultaneously modulated by a high-rate pseudo random bit sequence (PRBS). The modulation phase within each symbol is a value of either 0 or $\pi$ with equal probabilities, and moreover, the duration of each bit is much shorter than the acoustic wave lifetime. Within the correlation peak, the phase difference between the pump wave and probe wave is fixed, so that the acoustic wave is built up and kept at a steady state. In other positions, the acoustic wave is randomly alternating between the states of being excited and quenched, resulting in a zero expectation value. Similar to the frequency modulated BOCDA, the SBS interaction is only generated at the correlation peak. Recently, we proposed the phase-shift-keying BOCDA technique, which only generates two phase shifts $(0$ and $\pi)$ for phase coding using a Mach-Zehnder modulator (MZM) [14]. This phase coding technique is not sensitive to the bandwidth of the related devices. A PRBS phase coding with 20-ps bit duration without the transition edge is realized, obtaining a 2-mm spatial resolution, as shown in Fig. 5.

\subsection{Long-range distributed sensing}

When it comes to a long sensing range, due to the fiber attenuation, both pump pulse and $\mathrm{CW}$ probe powers need to be increased to get an adequate SNR over the entire sensing fiber, which can induce some nonlinear effects, including the Kerr effect [39, 40] and non-local effect [41-43], and consequently limits the extending of the sensing range. The power-dependent Kerr effect (including self-phase modulation SPM and cross-phase modulation XPM) and the fiber dispersion (group velocity dispersion, GVD) could deteriorate the system performance and reduce the sensing length. Usually, a long-range BOTDA system includes a 


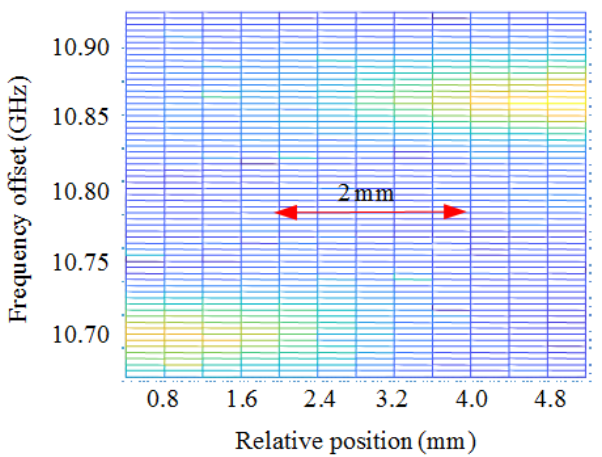

(a)

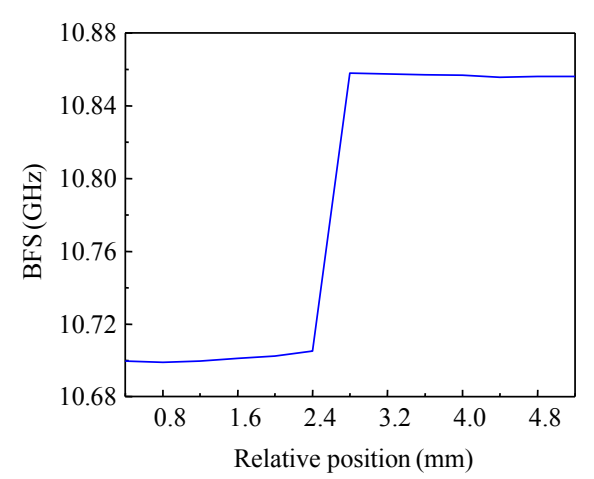

(b)

Fig. 5 Measured results of BOCAD: (a) measured Brillouin gain spectrum in the vicinity of the spliced joint of the fiber under test and (b) reconstructed local BFS [95].

high-power (hundreds of milliwatts) pump pulse and a low-power (tens of microwatts) $\mathrm{CW}$ probe wave. So the SPM and GVD of the CW probe can be neglected, meanwhile, the XPM can also be neglected due to the low-power probe. The pump pulse width is usually tens of nanoseconds, so that the effective of the GVD on the SPM is negligible. In either normal $\left(\beta_{2}>0\right)$ or anomalous dispersion $\left(\beta_{2}<0\right)$ fiber, the SPM will give rise to an intensity-dependent phase shift and a consequence spectrum broadening, while the pulse shape remains unaffected. The SPM induces a continuum spectral broadening, the extent of which depends on the pulse shape. Figure 6 shows the measured BGSs at the end of the $25-\mathrm{km}$ fibers at different pump pulse powers for Corning LEAF fiber (anomalous dispersion) and MetroCor fiber (normal dispersion). It is clear that the SPM can cause continuum spectrum broadening on a Gaussian pulse in both of anomalous and normal dispersion fibers at high powers [39].

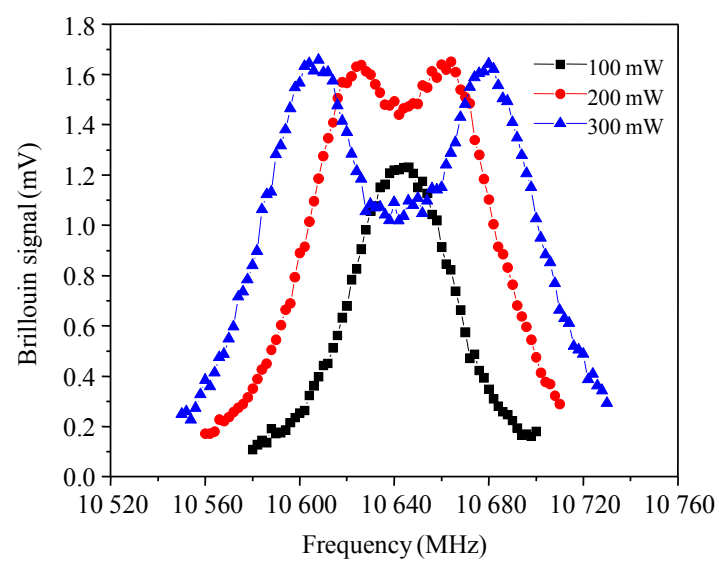

(a)

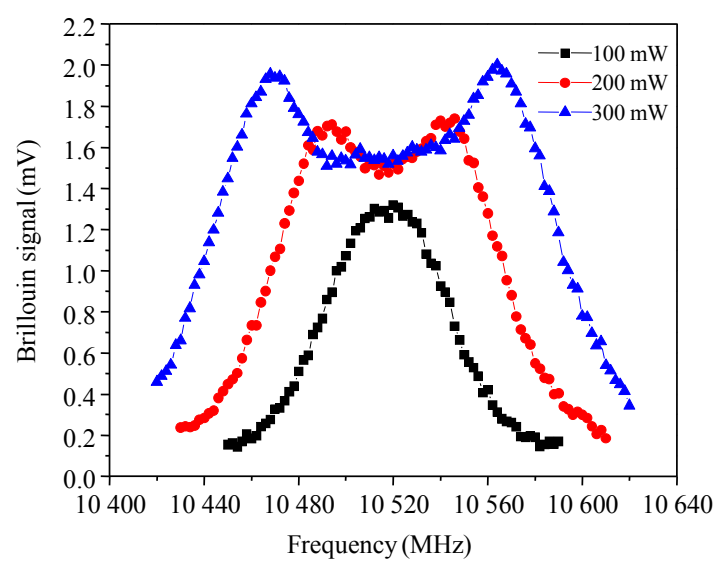

(b)

Fig. 6 Measured BGSs at the end of the $25-\mathrm{km}$ fibers with a 50-ns Gaussian pump pulse at different powers for (a) LEAF fiber (anomalous dispersion) and (b) MetroCor fiber (normal dispersion) [39].

However, for a square pulse, modulation instability (MI) could play an important role for its propagation. MI is a result of the interplay between the SPM and anomalous dispersion. MI occurs only in the anomalous dispersion regime $\left(\beta_{2}<0\right)$, and it will cause discrete spectral broadening through inducing additional discrete spectral lines. Figure 7(a) shows the measured 3-dimensional (3-D) BGSs with a $700-\mathrm{mW}$ pump pulse over a $25-\mathrm{km}$ LEAF fiber. The Brillouin signal exhibits an inflection point at the position of $\sim 8 \mathrm{~km}$, beyond which the signal decreases sharply. It is expected that the inflection point would appear in advance at a higher power. 
Note that the Brillouin signal features oscillation instead of monotone decreasing with the length, which is referred to as Fermi-Pasta-Ulam (FPU) recurrence in nonlinear systems [40]. It is also noted that the BGSs after the inflection point still keep a narrow linewidth as that in the front segment, which confirms that the MI effect only induces discrete spectrum broadening. The MI effect can be suppressed in a normal dispersion fiber such as MetroCor. Figure 7(b) shows the measured 3-D BGSs with a $700-\mathrm{mW}$ pump power over a $25-\mathrm{km}$ MetroCor fiber, where the Brillouin signal only shows smoothly decreasing only due to the fiber attenuation.

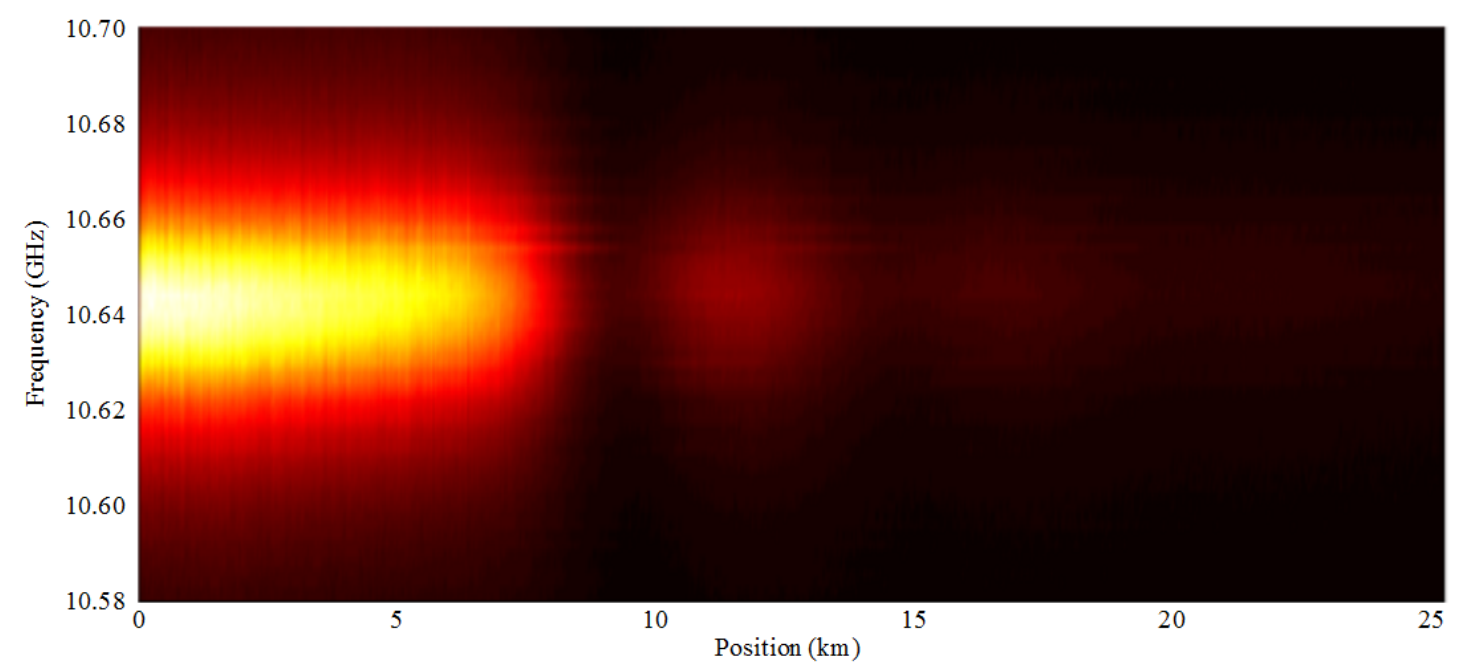

(a)

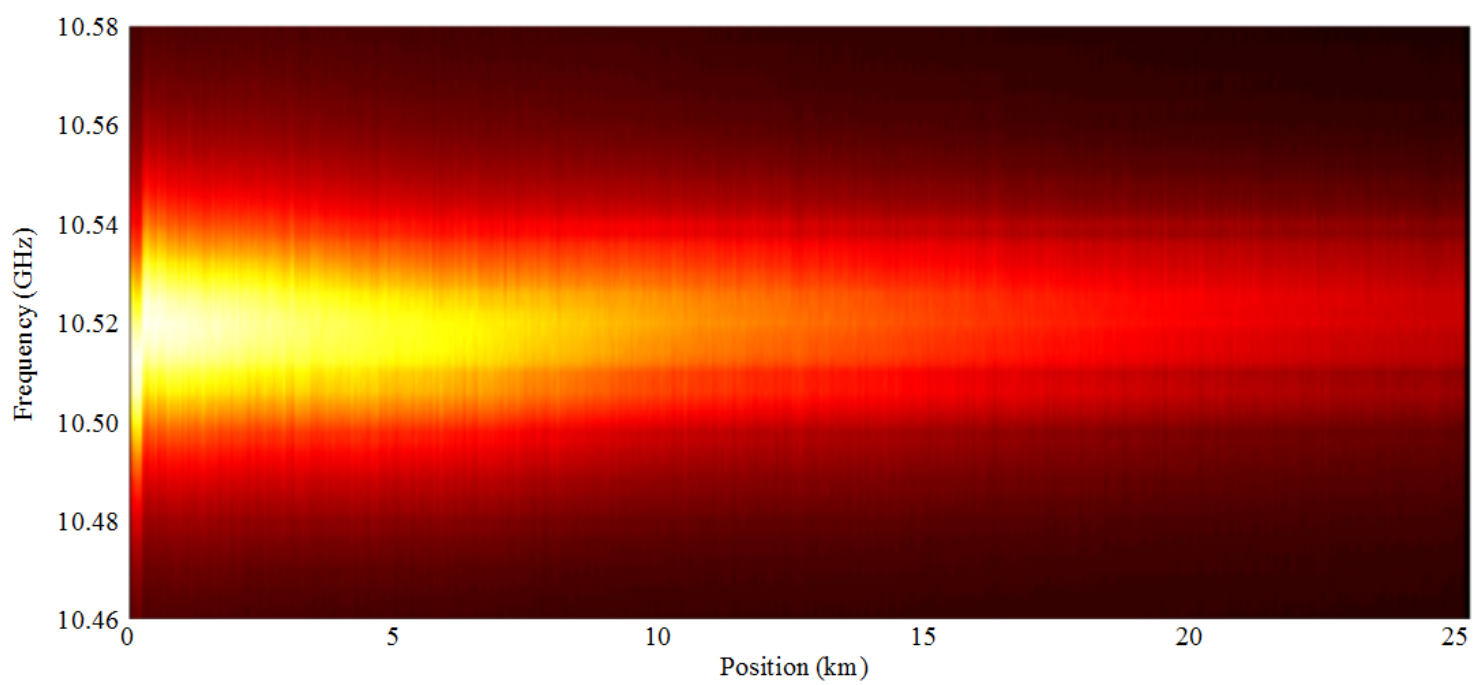

(b)

Fig. 7 Measure 3-D BGSs with a 50-ns square pump pulse at a power of $700 \mathrm{~mW}$ in (a) a $25-\mathrm{km}$ LEAF fiber (anomalous dispersion) and (b) a 25-km MetroCor fiber (normal dispersion) [39].

The non-local effect in a BOTDA system includes two cases, i.e., the pump pulse depletion in a Brillouin gain-based sensor and the probe pulse excessive amplification in a Brillouin loss-based sensor. Pump depletion refers to that when the frequency offset between the pump and probe waves equals to the BFS of the sensing fiber, a considerable portion of the pump power is transferred to the $\mathrm{CW}$ probe wave, which not only decreases the Brillouin gain but also makes the 
pump pulse power unequally depleted for different frequency offsets at the far end of the sensing fiber, resulting in distorted Brillouin gain spectra and systematic measurement errors. The pump depletion will be maximized for the worst case when the sensing fiber has a uniform BFS. Similarly, excess amplification in a Brillouin-loss based sensor can also lead to distorted Brillouin loss spectra and systematic measurement errors at the far end of the sensing fiber, and a distorted Brillouin loss spectrum induced by the nonlocal effect is shown in Fig. 8 [43].

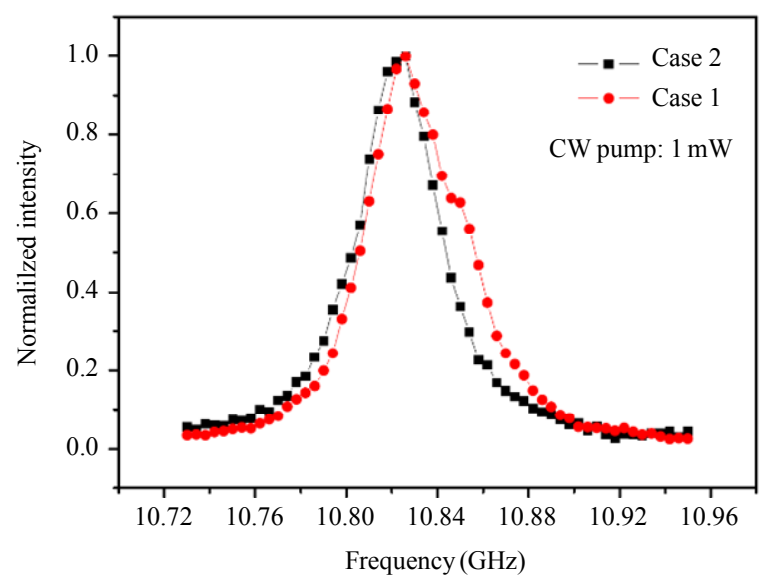

Fig. 8 Measured Brillouin loss spectra with the non-local effect (Case 1) and without non-local effect (Case 2) [43].

Time-division multiplexing and frequencydivision multiplexing BOTDA have been proposed to overcome the nonlocal effect and extend the sensing range [47]. In time-division multiplexing scheme, instead of a pulse and a CW wave as used in a conventional BOTDA, two pulses, i.e., a probe pulse and a pump pulse are used to perform the measurement. The spatial resolution is still defined by the duration of the narrow pump pulse, while the sensing length is determined by the duration of the long probe pulse. The delay between the pump pulse and probe pulse can be changed to select the sensing section where the probe pulse interacts with the pump pulse. The measurement of the entire sensing fiber is realized by implementing the measurement for each sensing section through changing the delay between the two pulses. Because the interaction length is determined by the duration of the probe pulse instead of the entire fiber, the probe power can be increased to enhance the Brillouin interaction in individual sections to improve the SNR without the non-local effect. A $100-\mathrm{km}$ sensing fiber is divided into 11 sections based on the gain-controlled principle, and spatial resolutions of $0.6 \mathrm{~m}$ and $2 \mathrm{~m}$ are obtained at the ends of $75 \mathrm{~km}$ and $100 \mathrm{~km}$, respectively [47].

The frequency-division multiplexing BOTDA features multiple sections with different BFSs in sensing fibers, so that the effective Brillouin interaction length is restricted to one resonant Brillouin frequency section rather than the entire sensing length, thus the non-local effect can be considerably reduced [48]. In addition, because the sensing fiber includes different types of fibers with different BFSs, the spontaneous Brillouin scattering cannot accumulate over the whole sensing fiber, which is another advantage of frequency-division multiplexing. A $150-\mathrm{km}$ sensing range is achieved by dividing the sensing fibers into two spans of equal length with in-line EDFAs placed between spans, and a $100 / 120 \mathrm{~ns}$ pulse pair is used to realize a $2-\mathrm{m}$ spatial resolution. At the end of the $150-\mathrm{km}$ sensing fiber, two 2-m stretched sections sandwiching a 2-m loose section can be clearly distinguished with each other, as shown in Fig. 9 [48].

\subsection{Fast distributed sensing}

For the classical BOTDA scheme, the temperature or strain measurement time is usually several seconds to several minutes long, which limits its applications to a static or a slowly-varying measurement. The expression of the maximum sampling rate can be written as [17]

$$
f_{s_{-} \text {BOTDA }}=\frac{1}{\left(T_{\text {round_trip }} \cdot N_{\text {code }} \cdot N_{\text {ave }}+T_{\text {switch }}\right) \cdot N_{f}}
$$

where $T_{\text {round _trip }}=2 n L / c$ is the round-trip time of the pump pulse, which is an inherent limitation related to the length of the sensing fiber $L . N_{\text {code }}$ is the code length of the pump pulse number. For 
instance, this value should be set to 2 for the DPP-BOTDA scheme while it depends on the encoding bits and rules for the coding approach. $N_{\text {ave }}$ is the time of the averaging. $T_{\text {switch }}$ is the frequency-switching time of the microwave generator (MWG). $N_{f}$ is the effective frequencysweeping number for the probe wave, which is used to measure the whole $\mathrm{BGS}$ and restrict the

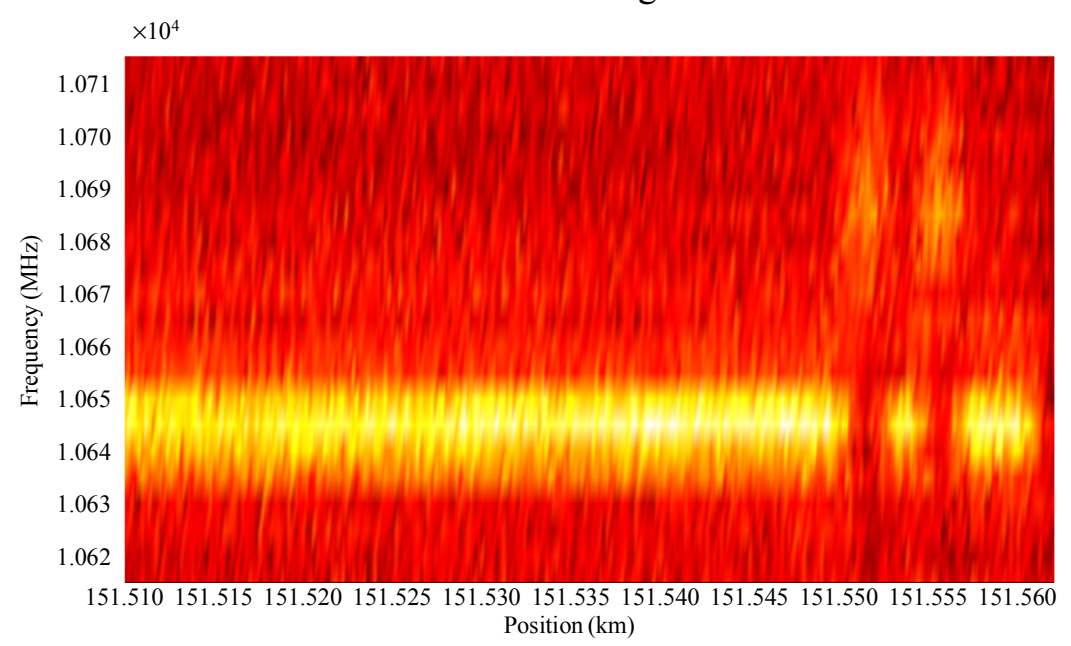

Fig. 9 Measured 3-D Brillouin spectra in the end of the 150-km sensing fiber [48].

To increase the sampling rate of the BOTDA scheme, the recent hotspots focused on reducing the $T_{\text {switch }}$ by using the high-performance electrical devices, decreasing the $N_{f}$ by using novel sensing curves.

To reduce $T_{\text {switch }}$, a technique based on the generation of the frequency-agility microwave signal by an electric arbitrary waveform generator (AWG) was proposed to realize fast BOTDA or F-BOTDA, where the fast-changing of the signal frequency is achieved by pre-writing the desired frequencies into the memory of the AWG [30]. This method features a fast measurement time and a broad measurement range. With 13-ns pump pulses, a spatial resolution of $1.3 \mathrm{~m}$ is realized over a $100-\mathrm{m}$ fiber observing a vibration frequency of up to $100 \mathrm{~Hz}$. The F-BOTDA faces the same problem as that in the conventional BOTDA in terms of spatial resolution, which is limited to $\sim 1 \mathrm{~m}$ by the 10 -ns phonon lifetime in silica fibers. We have proposed a modified DPP scheme, i.e., differential double-pulse, temperature/strain dynamic range. For the classical BOTDA, generally, $N_{\text {code }}=1, N_{\text {ave }}$ is several tens for a polarization-maintaining fiber and thousands for a standard SMF, the $T_{\text {switch }}$ is on the order of $\sim$ ms scale that is limited by the property of the MWG, and $N_{f}$ is usually $\sim 100$ for a frequency interval of several $\mathrm{MHz}$ to obtain a wide dynamic range of several hundreds of $\mathrm{MHz}$. for a high-spatial-resolution F-BOTDA. Instead of using a single pump pulse in a usual case, two long pulses with different pulse-widths are used to form a differential double-pulse used as pump pulses, so that the two Brillouin signals are obtained almost at the same time, which allows for fast acquisition of the differential signal for the high-spatial-resolution F-BOTDA, as shown in Fig. 10. In addition, we proposed to use the second-order sideband as the probe wave through appropriately adjusting the operation point of the modulator with the carrier and odd-order sidebands being suppressed and subsequently reducing the bandwidth requirement of the AWG by half to $\sim 5.5 \mathrm{GHz}$. A spatial resolution of $20 \mathrm{~cm}$ is achieved by using a 52/50 ns differential double-pulse, and the distributed vibration measurement is demonstrated over a 50-m Panda polarization-maintaining fiber observing the vibration frequency of up to $50 \mathrm{~Hz}$ [100]. To further decrease the bandwidth requirement for the AWG, a dual-modulation scheme [31] is proposed by 
employing a single-frequency modulation for a pump wave and a frequency-agility modulation for the probe wave, where a lower bandwidth of only several hundred of $\mathrm{MHz}$ for the $\mathrm{AWG}$ is required.

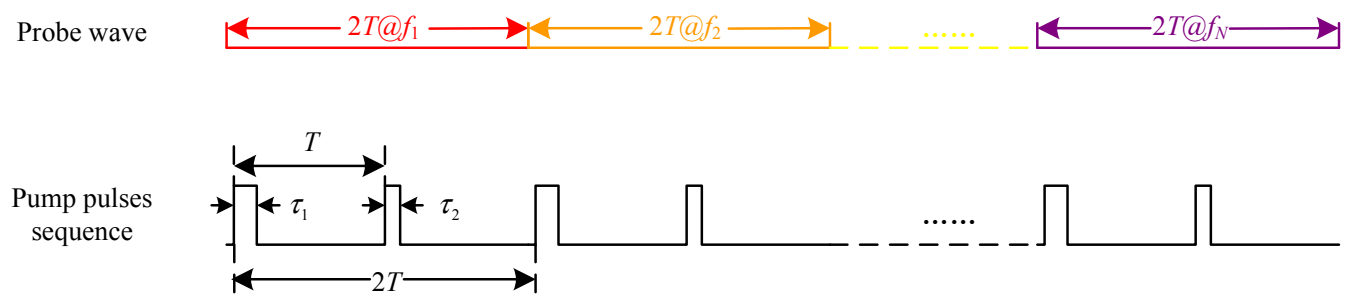

Fig. 10 Scheme of a high-spatial-resolution F-BOTDA [100].

A slope-assisted BOTDA for fast strain variations measurement has been proposed by only single pump pulse and using a single-slope of the local Brillouin gain spectrum [23, 24]. The maximum sampling rate is only determined by the fiber length and average number, but its measurable strain range is limited to the narrow-slope of BGS ( $\sim 30 \mathrm{MHz}$ for the single-mode fiber). A double slope-assisted BOTDA has been proposed to be immune to pump pulse power variations by using both side slopes of the BGS [25]. A multi-slope assisted fast BOTDA has been realized based on the double-slope demodulation and frequency-agile technique, which can extend the temperature/strain range by adding the frequency segments number of probe wave [26]. We also define a dimensionless coefficient $K$ as the ratio of Brillouin phase-shift to gain in the vector SBS effect. $K$ has a linear proportional relationship with the frequency detuning for the continuous waves, while it is insensitive to the power fluctuation of the pump pulse, as shown in Fig. 11. We have developed a slope-assisted BOTDA system based on the $K$ spectrum and frequency-agile technique, where it not only is immune to the variation of pump power but also can make full use of the frequency span of vector SBS. A multislope-assisted BOTDA is implemented for a dynamic measurement and a maximum strain variation up to $5372.9 \mu \varepsilon$ with a vibration frequency of $5.58 \mathrm{~Hz}$ is achieved [29].

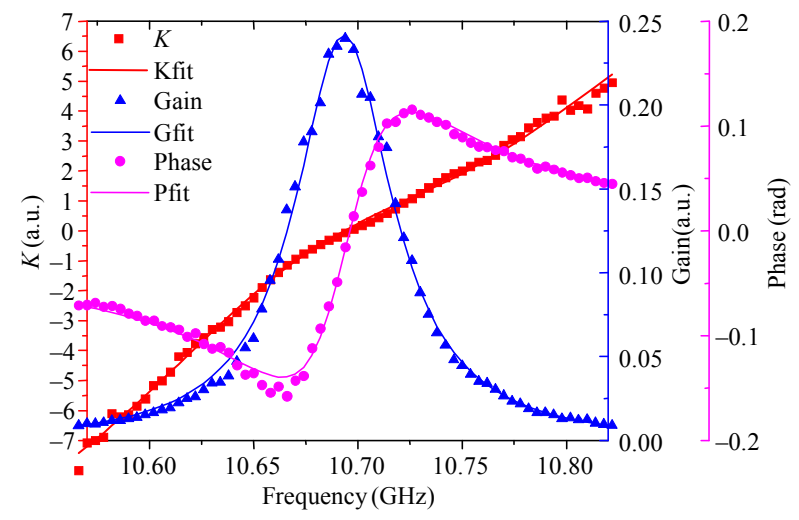

Fig. 11 Different spectra (gain, phase, and $K$ ) for a 20-ns pump pulse [29].

In 2018, we proposed a novel optical chirp chain (OCC) BOTDA scheme for distributed ultra-fast measurement, as shown in Fig. 12 [34]. The OCC probe wave is composed of several short optical chirp segments which are generated through frequency-modulation by the frequency-agile technique. When the frequency span between the OCC probe wave and the pump pulse overlaps the BFS of the sensing fiber, the distributed BGS along the fiber are illustrated via the OCC probe wave in the time (or position) domain with only single-shot pump pulse. The sampling rate of this proposed OCC-BOTDA is only restricted by the fiber length, and the maximum sampling rate can be up to $6.25 \mathrm{MHz}$ [34]. We also demonstrate a fast long-range BOTDA sensor by using the OCC technique and Brillouin loss scheme. The OCC probe wave with a broadband spectrum has been proved capable of increasing the SBS threshold, avoiding the non-local effect and amplifying the 
pump pulse. A sensing range of $150 \mathrm{~km}$ has been realized without any other amplification scheme, obtaining a spatial resolution of $6 \mathrm{~m}$ and a measurement time of $3.2 \mathrm{~s}$ [35]. However, OCC-BOTDA also has problems introduced by transient SBS, resulting in the broadened asymmetric Brillouin spectrum, the ghost peak, and the frequency shift of the main peak. A high-performance OCC-BOTDA using the principal component analysis and DPP technique is performed to overcome these problems, obtaining 4-m spatial resolution and 5-s measurement time over a $100-\mathrm{km}$ fiber [36]. Recently, a fast BOTDR has also been demonstrated by using the $\mathrm{OCC}$ as the reference wave, where the spontaneous Brillouin spectrum can be online demodulated in the time domain for truly distributed, one end access, and fast measurement. A sampling rate of $200 \mathrm{~Hz}$ is demonstrated over a 400-m single-mode fiber. Moreover, the combination of the OCC technique and BOTDR scheme enables the BGS with no distortion and ghost peak [37].

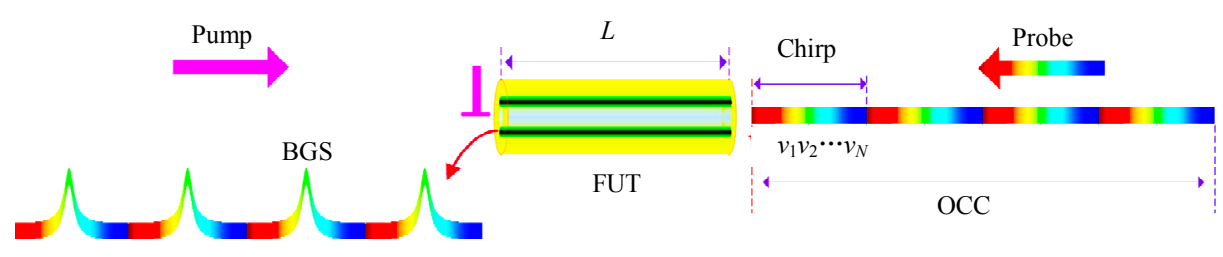

(a)
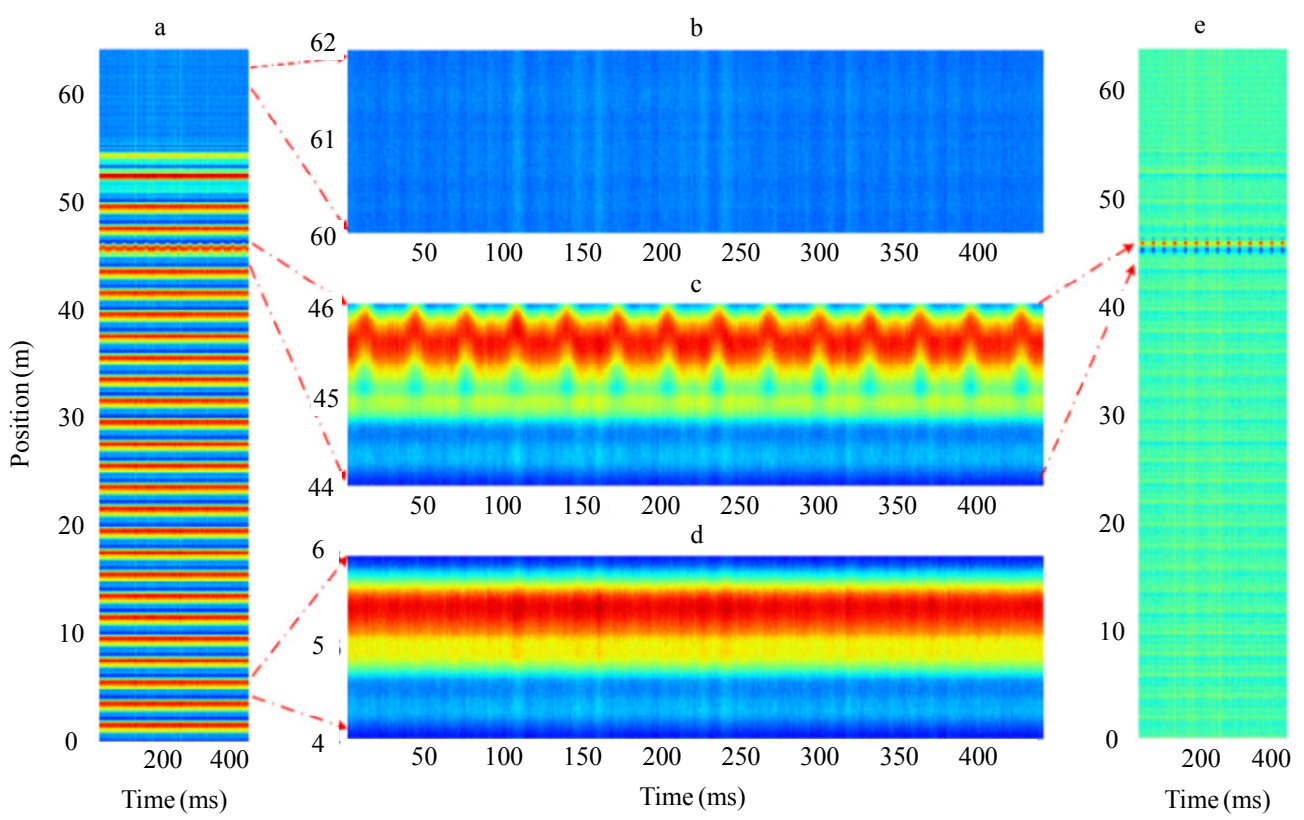

(b)

Fig. 12 OCC-BOTDA: (a) operation principle and (b) dynamic measurement [34].

\section{Distributed sensing based on Brillouin dynamic grating}

\subsection{Principle}

Generally, a Brillouin dynamic grating (BDG) is generated by using two counter-propagating pump waves through SBS. The BDG is similar to a conventional Fiber Bragg grating (FBG) in which both of them are local refractive-index-modulated grating structures. However, the BDG has two unique features: one is that it is a moving grating, which can produce a frequency shift (equal to BFS) 
on the reflected light with respect to the probe wave, and the other is that it has a lifetime $(\sim 10 \mathrm{~ns}$ for silica fiber) for its existence after removing the two pump waves.

For BDG applications, a polarizationmaintaining fiber (PMF) is usually used, where two pump waves are launched into one axis of the PMF to create a BDG and a probe wave is launched into the other axis to read the grating. When the frequency difference between the probe wave and pump waves satisfies the phase matching condition, a maximum reflection on the BDG can be observed. This process involves four light waves, i.e., two pump waves, a probe wave, and a reflected wave, and in fact it is a Brillouin enhanced four-wave mixing process. The interaction between the probe wave and the BDG involves two cases: one case is that the probe wave propagates in the same direction as the $\mathrm{BDG}$, the reflection of probe wave on the BDG is a coherent Stokes Brillouin scattering, and the frequency of the reflected wave is higher than that of the probe wave by a $\Omega_{B}$; the other case is for the counter-propagating between the probe wave and the BDG, thus it is a coherent anti-Stokes Brillouin scattering and the frequency of reflected wave is up-converted by a $\Omega_{B}$ with respective to the probe wave. The phase matching condition of the coherent Stokes Brillouin scattering or coherent anti-Stokes Brillouin scattering in a polarization-maintaining fiber is [60]

$$
\Delta v_{\text {Bire }}=\frac{\Delta n v}{n_{g}}
$$

where $\Delta n$ is the phase birefringence of the PMF, $n_{g}$ is the group refractive index, and $v$ is the frequency of the probe wave. $\Delta v_{\text {Bire }}$ is the birefringence-induced frequency shift between the probe and pump waves and is proportional to the local phase birefringence of the PMF. Figure 13 shows the schematic diagram of generation and detection of the BDG in a PMF.

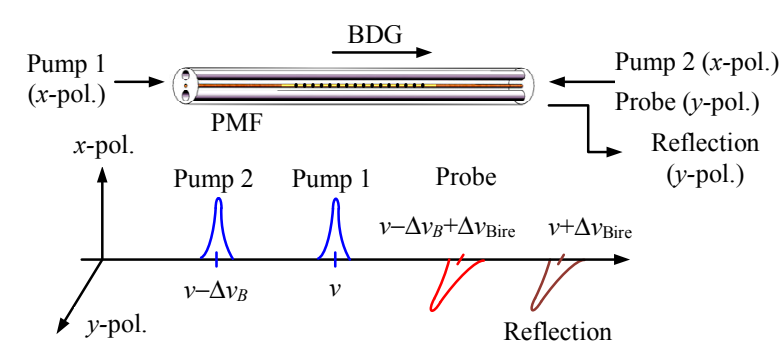

Fig. 13 Schematic diagram of generation and detection of the BDG in a PMF.

\subsection{Distributed multi-parameter sensing}

Brillouin scattering based distributed optical fiber sensors have shown the capability of distributed strain and temperature measurement. However, since both strain and temperature can produce a BFS change, the Brillouin based sensor suffers from the cross-sensitivity. Normally, it cannot be determined that a BFS change is induced by a change in temperature or strain. In a PMF, both temperature and strain can alter its birefringence, and it has been found that the birefringence-induced frequency shift of Panda PMF increases with the strain and decreases with temperature, which provides another uncorrelated mechanism to measure the temperature and strain change.

The dependences of the changes of Brillouin frequency shift and birefringence-induced frequency shift, $\Delta v_{B}$ and $\Delta v_{\text {Bire }}$, on strain and temperature can be expressed by

$$
\begin{aligned}
& \Delta \nu_{B}=C_{B}^{\varepsilon} \Delta \varepsilon+C_{B}^{T} \Delta T \\
& \Delta \nu_{\text {Bire }}=C_{\text {Bire }}^{\varepsilon} \Delta \varepsilon+C_{\text {Bire }}^{T} \Delta T
\end{aligned}
$$

where $\Delta \varepsilon$ and $\Delta T$ are changes in strain and temperature, respectively; $C_{B}^{\varepsilon}$ and $C_{B}^{T}$ are coefficients of strain and temperature related to the BFS, respectively; $C_{\text {Bire }}^{\varepsilon}$ and $C_{\text {Bire }}^{T}$ are coefficients of strain and temperature related to birefringence-induced frequency shift, respectively. The strain and the temperature can then be uniquely determined by the measured $\Delta v_{B}$ and $\Delta v_{\text {Bire }}$, and can be given by 


$$
\left[\begin{array}{c}
\Delta \varepsilon \\
\Delta T
\end{array}\right]=\frac{1}{C_{B}^{\varepsilon} C_{\text {Bire }}^{T}-C_{B}^{T} C_{\text {Bire }}^{\varepsilon}}\left[\begin{array}{cc}
C_{\text {Bire }}^{T} & -C_{B}^{T} \\
-C_{\text {Bire }}^{\varepsilon} & C_{B}^{\varepsilon}
\end{array}\right]\left[\begin{array}{c}
\Delta v_{B} \\
\Delta v_{\text {Bire }}
\end{array}\right]
$$

where $C_{B}^{\varepsilon}, C_{B}^{T}$, and $C_{\text {Bire }}^{\varepsilon}$ have positive sign, while $C_{\text {Bire }}^{T}$ has a negative sign, so $C_{B}^{\varepsilon} C_{\text {Bire }}^{T}-C_{B}^{T} C_{\text {Bire }}^{\varepsilon}$ is of a large value, which ensures a high discrimination accuracy. Usually, the temperature and strain coefficients of the BFS are $C_{B}^{T}=1.12 \mathrm{MHz} /{ }^{\circ} \mathrm{C}$ and $C_{B}^{\varepsilon}=0.0482 \mathrm{MHz} / \mu \varepsilon$, respectively. The dependences of birefringence-

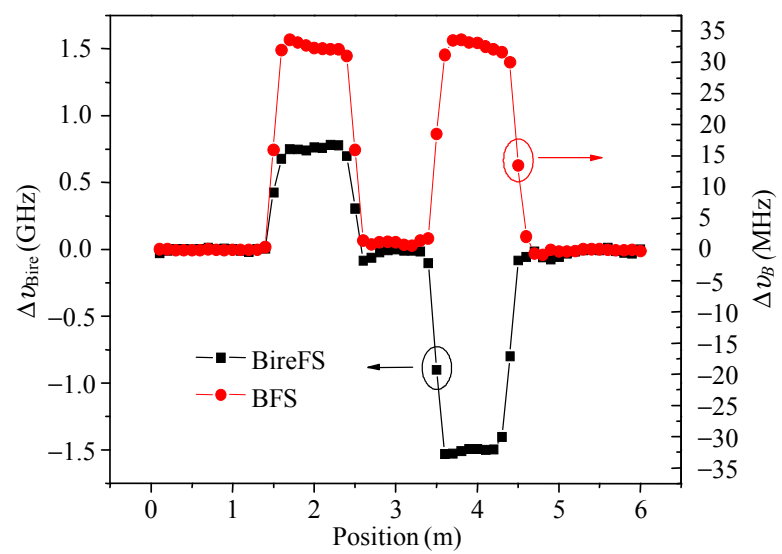

(a) induced frequency shift on temperature and strain of a Panda PMF are $C_{\text {Bire }}^{T}=-54.38 \mathrm{MHz} /{ }^{\circ} \mathrm{C}$ and $C_{\text {Bire }}^{\varepsilon}=1.13 \mathrm{MHz} / \mu \varepsilon$, respectively. Figure 14 shows the measured birefringence-induced frequency shift, Brillouin frequency shift, and the demodulated temperature and strain in an experiment [78]. Note that the BireFS provides higher precision with respect to BFS, so that the discrimination accuracy is limited by the uncertainty of BFS, where the temperature and strain accuracy is $0.4^{\circ} \mathrm{C}$ and $9 \mu \varepsilon$.

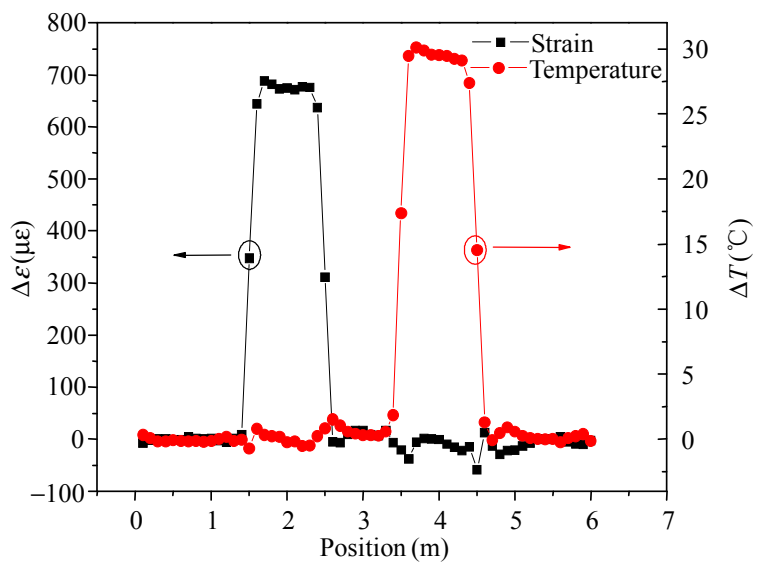

(b)

Fig. 14 Discrimination of temperature and strain: (a) measured birefringence-induced frequency shift and Brillouin frequency shift and (b) demodulated temperature and strain [78].

High-sensitivity distributed sensing of transverse load or hydrostatic pressure can also be realized based on the BDG, where the principle is to measure the birefringence change induced by the transverse load or hydrostatic pressure. Meanwhile, the temperature cross-talk to the transverse load or hydrostatic pressure can be compensated through measuring the temperature-induced BFS changes using Brillouin optical time-domain analysis. A distributed measurement of hydrostatic pressure is demonstrated experimentally using a 4-m sensing fiber with a maximum measurement error less than $0.03 \mathrm{MPa}$ at a 20-cm spatial resolution [75]. A distributed measurement of transverse load is demonstrated experimentally using a $10 \mathrm{~m}$ sensing fiber with a measurement accuracy as high as $0.8 \times 10^{-3} \mathrm{~N} / \mathrm{mm}$ at a $20 \mathrm{~cm}$ spatial resolution [74].

We have proposed and experimentally demonstrated a distributed salinity sensor based on the BDG with a PI-coated PM-PCF as the sensing component. The PI-coating will swell and shrink when in contact with solutions of different salinities. The swell and shrink responses induced by the change of the salinity are converted to the birefringence modulation loaded on the PM-PCF substrate. The salinity is then distributed measured by mapping the birefringence changes on the PM-PCF by the BDG. Four sensors with different coating thicknesses $(5 \mu \mathrm{m}, 8 \mu \mathrm{m}, 15 \mu \mathrm{m}$, and $20 \mu \mathrm{m})$ have been employed as demos to investigate the temperature dependence and salinity sensitivity. The obtained results show a linear response at each coating thickness, with $30.1 \mathrm{MHz} /(\mathrm{mol} / \mathrm{L})$ for $5 \mu \mathrm{m}$, $75.4 \mathrm{MHz} /(\mathrm{mol} / \mathrm{L})$ for $8 \mu \mathrm{m}, 119.2 \mathrm{MHz} /(\mathrm{mol} / \mathrm{L})$ for $15 \mu \mathrm{m}$, and $139.6 \mathrm{MHz} /(\mathrm{mol} / \mathrm{L})$ for $20 \mu \mathrm{m}$, respectively. The proposed sensor features the 
distributed measurement with a measurement uncertainty less than $10 \mathrm{MHz}$ (corresponding to the obtained maximum salinity accuracy of $0.072 \mathrm{~mol} / \mathrm{L}$ ) at a spatial resolution of $15 \mathrm{~cm}$. In Fig. 15, the black and red curves show the measured birefringence frequency shift distributions corresponding to the salinity of $0 \mathrm{~mol} / \mathrm{L}$ and $4 \mathrm{~mol} / \mathrm{L}$, respectively; blue curve is the subtraction results clearly distinguishing the two different locations corresponding to the salinity change [76].

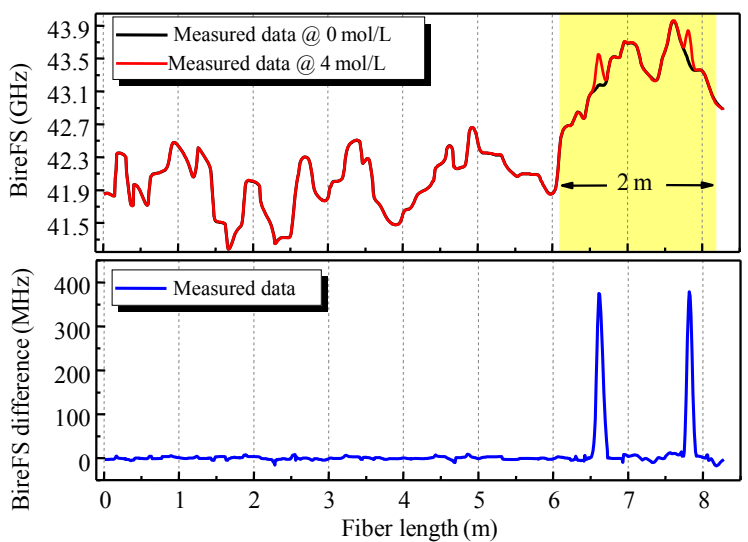

Fig. 15 Distributed measurement capability verification experiment with two salinity measuring segments in a $2-\mathrm{m}$ coated FUT [76].

\section{Distributed sensing based on forward stimulated Brillouin scattering}

\subsection{Principle}

The fiber not only acts as a waveguide for light, but also guides the acoustic wave propagation. Cylindrical single mode fiber can only support the fundamental light mode, while it can support abundant acoustic modes such as longitudinal, radial, torsional, and flexural elastic waves [90]. Whilst the optical mode is largely confined in the core of the fiber, having no chance to sense the fiber's surroundings directly, the existence of acoustic modes relies on the guidance of the acoustic waves by the cladding-exterior boundary, making it possible to perceive changes in the external mechanical properties of the surrounding medium. It should be noted that not all the acoustic modes, including longitudinal acoustic modes that resemble bulk acoustic waves, are sensitive to changes at the cladding-external medium boundary. However, transverse acoustic modes, which depend on the resonance across the fiber, are distinctly affected by any alteration of boundary. Acoustic waves can influence the propagation of light through the photo-elastic effect, which provides opportunities to identify components of surrounding materials by detecting light waves in standard fibers by the process of the FSBS. Radial acoustic modes denoted $R_{0, m}$ are used here, to represent a set of simplest transverse acoustic fields. The reflectivity coefficient of acoustic field magnitude at the boundary is determined by the external acoustic impedance and is expressed by

$$
r=\frac{\left|Z_{f}-Z\right|}{Z_{f}+Z} .
$$

The acoustic impedance equals the product of the material density and its acoustic velocity. $Z_{f}$ is the acoustic impedance of the silica fiber and $Z$ is the acoustic impedance of the material outside of the fiber.

The reflectivity coefficient of acoustic field magnitude at the boundary also affects the acoustic wave lifetime and the spectral linewidth of the FSBS. The quantitative relationship between the mechanical impedance of the external medium and FSBS spectrum is given by [85]

$$
\begin{gathered}
\Delta v=\Delta v_{i}+\Delta v_{b} \\
\Delta v_{b}=\frac{1}{\pi t_{r}} \ln \frac{Z+Z_{f}}{\left|Z-Z_{f}\right|}
\end{gathered}
$$

where $\Delta v$ denotes the total FSBS linewidth; $\Delta v_{i}$ is the intrinsic FSBS linewidth mainly determined by viscous damping of the acoustic wave; $\Delta v_{b}$ is the linewidth induced by the partial acoustic reflection at the boundary, which is determined by acoustic propagation time across the fiber diameter, $t_{r}$, the acoustic impedance of the exterior material, $Z$, and of silica, $Z_{f}$.

\subsection{Distributed acoustic impedance sensing}

The forward scattering characteristic renders 
FSBS sensor incapable of spatially-resolved distributed measurements. More recently, two remarkable works based on opto-mechanical time-domain reflectometry (OMTDR) [92] or local light phase recovery (LPR) [93] have been independently proposed to realize distributed FSBS resonance measurement for the first time, with a spatial resolution of $15 \mathrm{~m}-50 \mathrm{~m}$. Both schemes can be divided into three main stages. Firstly, some kind of FSBS interaction is used to sense the transverse acoustic information. Either OMTDR uses the energy transfer between two tones to read the acoustic wave, or else LPR utilizes the pre-excited acoustic vibration induced phase modulation of a single tone pulse. Secondly, one employs some technique to record the accumulated FSBS information. The backscattered light from the injected pulse is used to record the optical power at each position in either scheme, with the difference being that OMTDR uses the Rayleigh scattering while LPR uses stimulated Brillouin scattering. Finally, a demodulation algorithm based on differentiation is used to retrieve the local response of the transverse acoustic oscillation. The demodulation methods used in each scheme are determined by the nature of the FSBS interaction.

The spatial resolution of the distributed FSBS sensor comes from two sources. One is using pulses' backscattered light to record the evolution of optical power, so that each measurement point contains information about the state of the adjacent fiber. The other spatial resolution-determining factor is the data-processing. Due to the sensitivity to noise of the operation of differentiation, data smoothing is essential for the demodulation of a distributed FSBS spectrum, which inevitably degrades the spatial resolution. The SNR of the original data determines the smoothing window length, which is then closely associated with the spatial resolution. In the OMTDR method, the use of a long pulse required by a steady FSBS interaction, as well as the low-SNR detection method using weak Rayleigh scattering, restricts the further improvement of spatial resolution. In the LPR method, although the separation of activating and probing process permits the employment of a short reading pulse, the single tone probing only applies phase modulation to the probe light, which has a relatively weak energy transfer efficiency between the carrier and sideband frequencies. Therefore, it has relatively low SNR and thus restricts high spatial resolution sensing. The current spatial resolution, fundamentally limited by the noise level of the scattered light, poses a restriction on the practical applicability.

In 2020, we proposed a novel method named opto-mechanical time-domain analysis (OMTDA) based on coherent stimulated probing to remarkably improve the performance of a distributed FSBS sensor, as shown in Fig. 16 [94]. Firstly, a dual-tone long activation pulse and short probe pulse are launched sequentially into the fiber, where the beat frequency between two tones within each of the two pulses are equal. The transverse acoustic wave is excited by the activation pulse to create a stable state, which enhances the energy transfer between the two tones of the probe pulse and is subsequently detected. The blue part of the fiber indicates a different external material compared with rest of the fiber. Secondly, the low-power CW probe is injected into the fiber from the far end to map the intensity evolution of the two corresponding tones of the probe pulse [orange and violet lines in Fig. 16(a)] via BSBS, where the frequency of the $\mathrm{CW}$ probe is swept to get BOTDA signals of the two tones. Thirdly, the BOTDA signals are integrated over frequency to get smoother intensity traces $P_{1}(z)$ and $P_{2}(z)$. Fourthly, the two traces measured are processed mainly by division and differentiation to get the FSBS strength at each frequency. In the end, the FSBS frequency, $\Omega$, is swept and the measurement procedure is repeated to obtain the FSBS spectrum as a function of the distance along the fiber. 


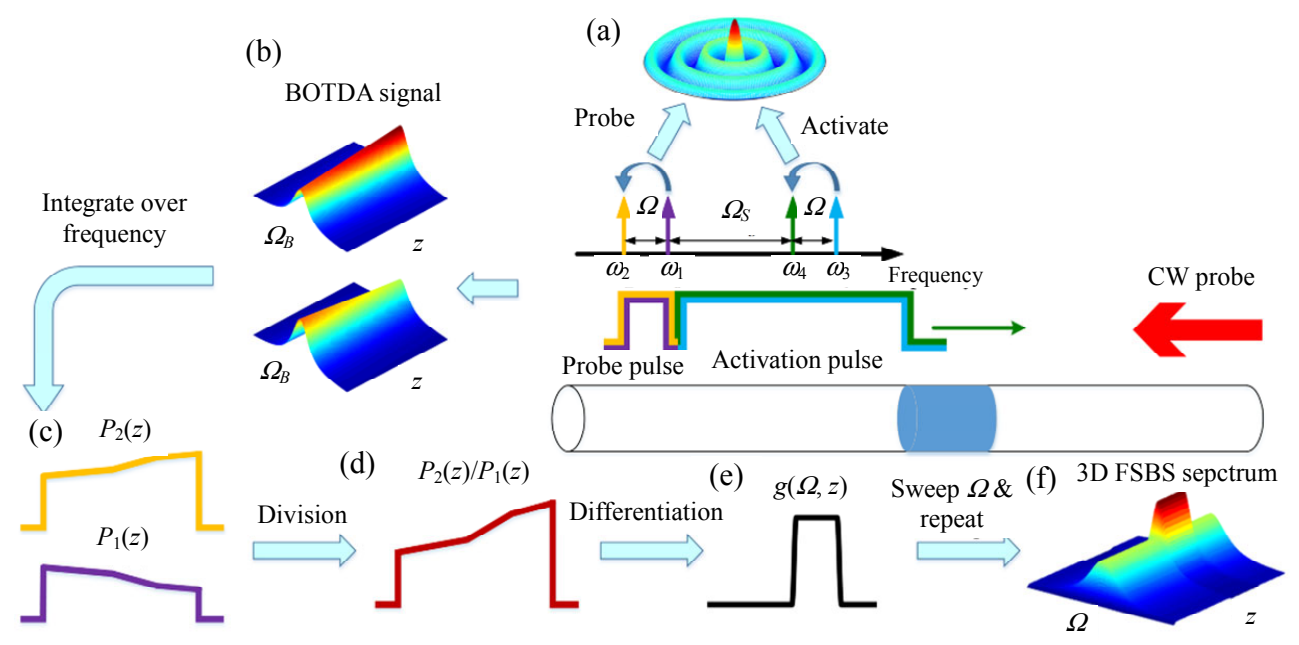

Fig. 16 Schematic diagram of the OMTDA [94].

Thanks to the proposed OMTDA scheme and our efforts to improve the SNR, we experimentally demonstrate a distributed measurement with $2 \mathrm{~m}$ spatial resolution for identification of air and alcohol, as shown in Fig. 17. Figure 17(a) shows the distributed spectral width of the FSBS (black line)

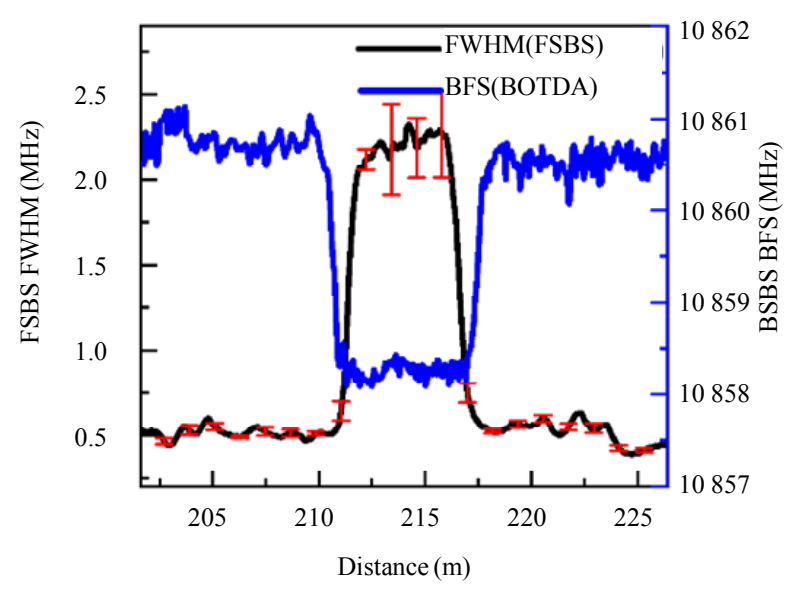

(a) and Brillouin frequency shift (BFS) of the BOTDA signal (blue line) for the uncoated fiber. Figure 17(b) shows the spectra of the FSBS for the fiber surrounded by alcohol (red dots) and air (black squares) with the spectral widths of $2.21 \mathrm{MHz}$ and $0.45 \mathrm{MHz}$, respectively.

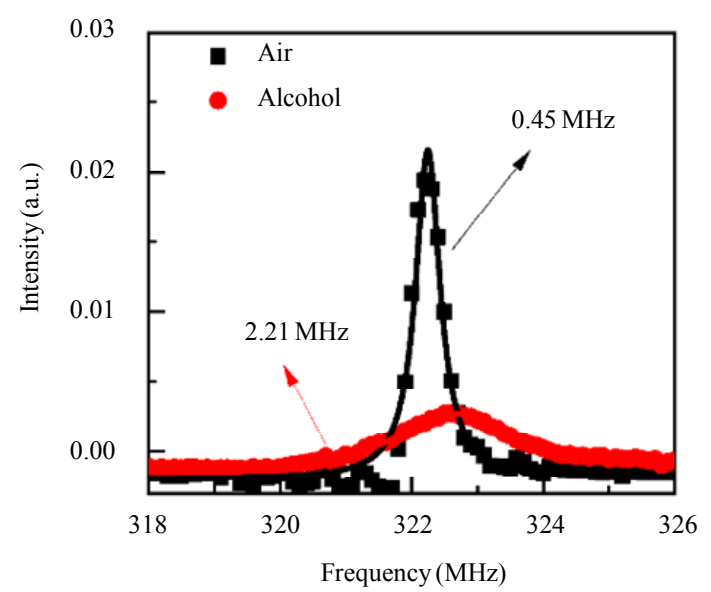

(b)

Fig. 17 Position-resolved substance identification with FSBS: (a) measured linewidth of FSBS and (b) measured FSBS spectra [89].

\section{Conclusions}

After three decades of development, the performance of distributed optical fiber sensors based on backward stimulated Brillouin scattering has gained huge advances in the aspects of spatial resolution, measurement time, and sensing range; meanwhile, they have found many applications in the fields of oil/gas industry, power industry, civil infrastructures and so on. On one hand, various techniques developed in optical communication can be introduced into distributed sensing to further 
improve their performance; on the other hand, machine learning can provide another dimension to improve their performance. Brillouin dynamic gratings and forward stimulated Brillouin scattering are two novel sensing mechanisms to realize distributed sensing, and they expand the measurable parameters from temperature/strain to salinity, static pressure, transverse pressure, and substance identification. In the future, the performance of these two novel distributed sensors could be further elevated from the aspects of both hardware and software. The spatial resolution of OMTDR/OMTDA is still restricted to meters, and a higher spatial resolution is required to expand its applications.

\section{Acknowledgment}

This work was funded by the National Key Scientific Instrument and Equipment Development Project of China (Grant No. 2017YFF0108700).

Open Access This article is distributed under the terms of the Creative Commons Attribution 4.0 International License (http://creativecommons.org/licenses/by/4.0/), which permits unrestricted use, distribution, and reproduction in any medium, provided you give appropriate credit to the original author(s) and the source, provide a link to the Creative Commons license, and indicate if changes were made.

\section{References}

[1] A. Barrias, J. R. Casaas, and S. Villalba, "A review of distributed optical fiber sensors for civil engineering applications," Sensors, 2016, 16(5): 748.

[2] X. Bao and L. Chen, "Recent progress in Brillouin scattering based fiber sensors," Sensors, 2011, 11(4): 4152-4187.

[3] S. Diakaridia, Y. Pan, P. Xu, D. Zhou, B. Wang, L. Teng, et al., "Detecting cm-scale hot spot over 24-km-long single-mode fiber by using differential pulse pair BOTDA based on double-peak spectrum," Optics Express, 2017, 25(15): 17727-17736.

[4] K. Hotate and M. Tanaka, "Distributed fiber Brillouin strain sensing with 1-cm spatial resolution by correlation-based continuous-wave technique," IEEE Photonics Technology Letters, 2002, 14(2): 179-181.
[5] X. Bao, A. Brown, M. DeMerchant, and J. Smith, "Characterization of the Brillouin-loss spectrum of single-mode fibers by use of very short $(<10-n s)$ pulses," Optics Letters, 1999, 24(8): 510.

[6] A. W. Brown, B. G. Colpitts, and K. Brown, "Distributed sensor based on dark-pulse Brillouin scattering," IEEE Photonics Technology Letters, 17(7): 1501-1503.

[7] K. Kishida, C. Li, and Nishiguchi, "Pulse pre-pump method for cm-order spatial resolution of BOTDA," SPIE, 5855: 559-562.

[8] W. Li, X. Bao, Y. Li, and L. Chen, "Differential pulse-width pair BOTDA for high spatial resolution sensing," Optics Express, 2008, 16(26): 21616-21625.

[9] Y. Dong, H. Zhang, L. Chen, and X. Bao, " $2 \mathrm{~cm}$ spatial-resolution and $2 \mathrm{~km}$ range Brillouin optical fiber sensor using a transient differential pulse pair," Applied Optics, 2012, 51(9): 1229-1235.

[10] A. Dominguez-Lopez, M. A. Soto, S. Martin-Lopez, L. Thevenaz, and M. Gonzalez-Herraez, "Resolving 1 million sensing points in an optimized differential time-domain Brillouin sensor," Optics Letters, 2017, 42(10): 1903.

[11] S. M. Foaleng, M. Tur, J. C. Beugnot, and L. Thevenaz, "High spatial and spectral resolution long-range sensing using brillouin echoes," Journal of Lightwave Technology, 2011, 28(20): 2993-3003.

[12] A. Zadok, Y. Antman, N. Primerov, A. Denisov, J. Sancho, and L. Thevenaz, "Random-access distributed fiber sensing laser," Laser \& Photonics Reviews, 2012, 6(5): L1-L5.

[13] R. Cohen, Y. London, Y. Antman, and A. Zadok, "Brillouin optical correlation domain analysis with 4 millimeter resolution based on amplified spontaneous emission," Optics Express, 2014, 22(10): 12070-12078.

[14] D. Ba, Y. Li, X. Zhang, J. Yan, and Y. Dong, "Phase-coded Brillouin optical correlation domain analysis with 2-mm resolution based on phase-shift keying," Optics Express, 2019, 27(25): 36197.

[15] J. Zhang, C. Feng, M. Zhang, Y. Liu, C. Wu, and Y. Wang, "Brillouin optical correlation domain analysis based on chaotic laser with suppressed time delay signature," Optics Express, 2018, 26(6): 6962-6972.

[16] K. Y. Song, Z. He, and K. Hotate, "Distributed strain measurement with millimeter-order spatial resolution based on brillouin optical correlation domain analysis and beat lock-in detection scheme," in 18th International Conference Optical Fiber Sensors, Mexico, 2006, ThC2.

[17] H. Zhang, D. Zhou, B. Wang, C. Pang, P. Xu, T. Jiang, et al., "Recent progress in fast distributed Brillouin optical fiber sensing," Applied Science, 2018, 8: 1820.

[18] C. Jin, N. Guo, Y. Feng, L. Wang, H. Liang, J. Li, et al., "Scanning-free BOTDA based on ultra-fine 
digital optical frequency comb," Optics Express, 2015, 23(4): 5277-5284.

[19] J. Fang, P. Xu, Y. Dong, and W. Shieh, "Single-shot distributed Brillouin optical time domain analyzer," Optics Express, 2017, 25(13): 15188-15198.

[20] D. Zhou, Y. Dong, and J. Yao, "Truly distributed and ultra-fast microwave photonic fiber-optic sensor," Journal of Lightwave Technology, 2020, 38(15): 4150-4159.

[21] C. Jin, L. Wang, Y. Chen, N. Guo, W. Chung, H. Au, et al., "Single-measurement digital optical frequency comb based phase-detection Brillouin optical time domain analyzer," Optics Express, 2017, 25(8): 9213-9224.

[22] Z. Liang, J. Pan, S. Gao, Q. Sui, Y. Feng, F. Li, et al., "Spatial resolution improvement of single-shot digital optical frequency comb-based Brillouin optical time domain analysis utilizing multiple pump pulses," Optics Letters, 2018, 43(15): 3534-3537.

[23] R. Bernini, A. Minardo, and L. Zeni, "Dynamic strain measurement in optical fibers by stimulated Brillouin scattering," Optics Letters, 2009, 34(17): 2613-2615.

[24] Y. Peled, A. Motil, L. Yaron, and M. Tur, "Slope-assisted fast distributed sensing in optical fibers with arbitrary Brillouin profile," Optics Express, 2011, 19(21): 19845-19854.

[25] A. Motil, O. Danon, Y. Peled, and M. Tur, "Pump-power-independent double slope-assisted distributed and fast Brillouin fiber-optic sensor," IEEE Photonics Technology Letters, 2014, 26(8): 797-800.

[26] D. Ba, B. Wang, D. Zhou, M. Yin, Y. Dong, H. Li, et al., "Distributed measurement of dynamic strain based on multi-slope assisted fast BOTDA," Optics Express, 2016, 24(9): 9781-9793.

[27] X. B. Tu, H. Luo, Q. Sun, X. Y. Hu, and Z. Meng, "Performance analysis of slope-assisted dynamic BOTDA based on Brillouin gain or phase-shift in optical fibers," Journal of Optics, 2015, 17(10): 105503.

[28] J. Urricelqui, A. Zornoza, M. Sagues, and A. Loayssa, "Dynamic BOTDA measurements based on Brillouin phase-shift and RF demodulation," Optics Express, 2012, 20(24): 26942-26949.

[29] D. Zhou, Y. Dong, B. Wang, T. Jiang, D. Ba, P. Xu, et al., "Slope-assisted BOTDA based on vector SBS and frequency-agile technique for wide-strain-range dynamic measurements," Optics Express, 2017, 25(3): 1889-1902.

[30] Y. Peled, A. Motil, and M. Tur, "Fast Brillouin optical time domain analysis for dynamic sensing," Optics Express, 2012, 20(8): 8584-8591.

[31] D. Ba, D. Zhou, B. Wang, Z. Lu, Z. Fan, Y. Dong, et al., "Dynamic distributed Brillouin optical fiber sensing based on dual-modulation by combining single frequency modulation and frequency-agility modulation," IEEE Photonics Technology Letters, 2017, 9(3): 1-8.

[32] Q. Chu, B. Wang, H. Wang, D. Ba, and Y. Dong, "Fast Brillouin optical time-domain analysis using frequency-agile and compressed sensing," Optics Letters, 2020, 45(15): 4365-4368.

[33] B. Wang, Z. Hua, C. Pang, D. Zhou, D. Ba, D. Lin, et al., "Fast Brillouin optical time-domain reflectometry based on the frequency-agile technique," Journal of Lightwave Technology, 2020, 38(4): 946-952.

[34] D. Zhou, Y. Dong, B. Wang, C. Pang, D. Ba, H. Zhang, et al., "Single-shot BOTDA based on an optical chirp chain probe wave for distributed ultrafast measurement," Light: Science \& Applications, 2018, 7(1): 32.

[35] Y. Dong, B. Wang, C. Pang, D. Zhou, D. Ba, H. Zhang, et al., " $150 \mathrm{~km}$ fast BOTDA based on the optical chirp chain probe wave and Brillouin loss scheme," Optics Letters, 2018, 43(19): 4679-4682.

[36] B. Wang, B. Fan, D. Zhou, C. Pang, Y. Li, D. Ba, et al., "High-performance optical chirp chain BOTDA by using a pattern recognition algorithm and the differential pulse-width pair technique," Photonics Research, 2019, 7(6): 652-658.

[37] D. Ba, B. Wang, T. Li, Y. Li, D. Zhou, and Y. Dong, "Fast Brillouin optical time-domain reflectometry using the optical chirp chain reference wave," Optics Letters, 2020, 45(19): 5460-5463.

[38] J. Zhang, H. Zheng, H. Wu, N. Guo, G. Yin, and T. Zhu, "Vector optical-chirp-chain Brillouin optical time-domain analyzer based on complex principal component analysis," Optics Express, 2020, 28(20): 28831-28842.

[39] Y. Dong and X. Bao, "Impacts of Kerr effect and fiber dispersion on long-range Brillouin optical timedomain analysis systems," in International Conference on Optical Fiber Sensor, China, 2012, pp. 84219Z-1-84219Z-4.

[40] K. Tai, A. Hasegawa, and A. Tomita, "Observation of modulational instability in optical fibers," Physical Review Letters, 1986, 56(2): 135.

[41] A. Dominguez-Lopez, X. Angulo-Vinuesa, A. Lopez-Gil, S. Martin-Lopez, and M. GonzalezHerraez, "Non-local effects in dual-probe-sideband Brillouin optical time domain analysis," Optics Express, 2015, 23(8): 10341-10352.

[42] L. Thevenaz, S. F. Mafang, and J. Lin, "Effect of pulse depletion in a Brillouin optical time-domain analysis system," Optics Express, 2013, 21(12): 14017-14035.

[43] Y. Dong, L. Chen, and X. Bao, "System optimization of a long-range Brillouin-loss-based distributed fiber sensor," Applied Optics, 2010, 49(27): 5020-5025.

[44] X. H. Jia, Y. J. Rao, L. Chen, C. Zhang, and Z. L. Ran, "Enhanced sensing performance in long 
distance Brillouin optical time-domain analyzer based on Raman amplification: theoretical and experimental investigation," Journal of Lightwave Technology, 2010, 28: 1624-1630.

[45] M. A. Soto, G. Bolognini, and F. D. Pasquale, "Optimization of long range BOTDA sensors with high resolution using first-order bi-directional Raman amplification," Optics Express, 2011, 19(5): 4444-4457.

[46] X. Angulo-Vinuesa, S. Martin-Lopez, J. Nuño, P. Corredera, J. D. Aniacastañon, L. Thévenaz, et al., "Raman-assisted Brillouin distributed temperature sensor over $100 \mathrm{~km}$ featuring $2 \mathrm{~m}$ resolution and $1.2{ }^{\circ} \mathrm{C}$ uncertainty," Journal of Lightwave Technology, 2012, 30(8):1060-1065.

[47] Y. Dong, L. Chen, and X. Bao, "Time-division multiplexing-based BOTDA over $100 \mathrm{~km}$ sensing length," Optics Letters, 2011, 36(2): 277-279.

[48] Y. Dong, L. Chen, and X. Bao, "Extending the sensing range of Brillouin optical time-domain analysis combining frequency-division multiplexing and in-line EDFAs," Journal of Lightwave Technology, 2012, 30(8): 1161-1167.

[49] A. M. Soto, G. Bolognini, F. D. Pasquale, and L. Thévenaz, "Simplex-coded BOTDA fiber sensor with $1 \mathrm{~m}$ spatial resolution over a $50 \mathrm{~km}$ range," Optics Letters, 2010, 35(2): 259-261.

[50] A. M. Soto, G. Bolognini, and F. D. Pasquale, "Long-range simplex-coded BOTDA sensor over $120 \mathrm{~km}$ distance employing optical preamplification," Optical Letters, 2011, 36(2): 232-234.

[51] A. M. Soto, M. Taki, and G. Bolognini, "Simplexcoded BOTDA sensor over 120-km SMF with 1-m spatial resolution assisted by optimized bidirectional Raman amplification," IEEE Photonics Technology Letters, 2012, 24(20): 1823-1826.

[52] J. J. Mompó, J. Urricelqui, and A. Loayssa, "Brillouin optical time-domain analysis sensor with pump pulse amplification," Optics Express, 2016, 24(12): 12672-12681.

[53] M. A. Soto, J. A. Ramírez, and L. Thévenaz, "Intensifying the response of distributed optical fibre sensors using 2D and 3D image restoration," Nature Communications, 2016, 7(1): 10870.

[54] S. Le Floch, F. Sauser, M. Llera and E. Rochat, "Novel Brillouin optical time-domain analyzer for extreme sensing range using high-power flat frequency-coded pump pulses," Journal of Lightwave Technology, 2014, 33(12): 2623-2627.

[55] X. H. Jia, H. Q. Chang, K. Lin, C. Xu, and J. G. Wu, "Frequency-comb-based BOTDA sensors for high-spatial-resolution/long-distance sensing," Optics Express, 2017, 25(6): 6997-7007.

[56] M. A. Soto, J. A. Ramírez, and L. Thévenaz, "Optimizing image denoising for long-range
Brillouin distributed fiber sensing," Journal of Lightwave Technology, 2017, 36(4): 1168-1177.

[57] B. Wang, L. Wang, C. Yu, and C. Lu, "Long-distance BOTDA sensing systems using video-BM3D denoising for both static and slowly varying environment," Optics Express, 2019, 27(25): 36100-36113.

[58] X. Sun, Z. Yang, X. Hong, S. Zaslawski, S. Wang, M. A. Soto, and L. Thévenaz, "Genetic-optimised aperiodic code for distributed optical fibre sensors," Nature Communications, 2020, 11(1): 1-11.

[59] Z. Zhu, D. J. Gauthier, and R. W. Boyd, “ Stored light in an optical fiber via stimulated Brillouin scattering," Science, 2007, 318(5857): 1748-1750.

[60] Y. Dong, L. Chen, and X. Bao, "Truly distributed birefringence measurement of polarizationmaintaining fibers based on transient Brillouin grating," Optics Letters, 2010, 35(2): 193-195.

[61] Y. Dong, H. Zhang, Z. Lu, L. Chen, and X. Bao, "Long-Range and high-spatial-resolution distributed birefringence measurement of a polarizationmaintaining fiber based on Brillouin dynamic grating," Journal of Lightwave Technology, 2013, 31(16): 2681-2686.

[62] Y. H. Kim and K. Y. Song, "Mapping of intermodal beat length distribution in an elliptical-core two-mode fiber based on Brillouin dynamic grating," Optics Express, 2014, 22: 17292-17302.

[63] Y. H. Kim and K. Y. Song, "Characterization of nonlinear temperature dependence of Brillouin dynamic grating spectra in polarization-maintaining fibers," Journal of Lightwave Technology, 2015, 33(23): 4922-4927.

[64] A. Li, Q. Hu, X. Chen, B. Y. Kim, and W. Shieh, "Characterization of distributed modal birefringence in a few-mode fiber based on Brillouin dynamic grating," Optical Letters, 2014, 39: 3153-3156.

[65] T. Jiang, D. Zhou, M. Xia, L. Teng, D. Ba, and Y. Dong, "Distributed birefringence measurement of a polarization-maintaining fiber with an extended range based on an enhanced Brillouin dynamic grating," IEEE Photonics Journal, 2020, 12(4): 7102507.

[66] V. P. Kalosha, W. Li, F. Wang, L. Chen, and X. Bao, "Frequency-shifted light storage via stimulated Brillouin scattering in optical fibers," Optical Letters, 2008, 33: 2848-2850.

[67] K. Y. Song, K. Lee, and S. B. Lee, "Tunable optical delays based on Brillouin dynamic grating in optical fibers," Optics Express, 2009, 17(12): 10344-10349.

[68] S. Chin and L. Thevenaz, "Tunable photonic delay lines in optical fibers," Laser \& Photonics Reviews, 2012, 6(6): 724-738.

[69] M. Santagiustina, S. Chin, N. Primerov, L. Ursini, and L. Thevenaz, "All-optical signal processing using dynamic Brillouin gratings," Scientific Reports, 
2013, 3: 1594.

[70] J. Sancho, N. Primerov, S. Chin, Y. Antman, A. Zadok, S. Sales, et al., "Tunable and reconfigurable multi-tap microwave photonic filter based on dynamic Brillouin gratings in fibers," Optics Express, 2012, 20: 6157-6162.

[71] L. Ursini and M. Santagiustina, "Applications of the dynamic Brillouin gratings to ultrawideband communications," IEEE Photonics Technology Letters, 25(14): 1347-1349.

[72] J. J. Guo, M. Li, Y. Deng, N. Huang, J. Liu, and N. Zhu, "Multichannel optical filters with an ultranarrow bandwidth based on sampled Brillouin dynamic gratings," Optics Express, 2014, 22(4): 4290-4300.

[73] Y. Dong, T. Jiang, L. Teng, H. Zhang, L. Chen, and $\mathrm{X}$. Bao, "Sub-MHz ultrahigh-resolution optical spectrometry based on Brillouin dynamic gratings," Optics Letters, 2014, 39(10): 2967-2970.

[74] Y. Dong, L. Teng, P. Tong, T. Jiang, H. Zhang, T. Zhu, et al., "High-sensitivity distributed transverse load sensor with an elliptical-core fiber based on Brillouin dynamic gratings," Optical Letters, 2015, 40(21): 5003-5006.

[75] L. Teng, H. Zhang, Y. Dong, D. Zhou, T. Jiang, W. Gao, et al., "Temperature-compensated distributed hydrostatic pressure sensor with a thin-diameter polarization- maintaining photonics crystal fiber based on Brillouin dynamic gratings," Optical Letters, 2016, 41(18): 4413-4416.

[76] H. Zhang, L. Teng, and Y. Dong, "Distributed salinity sensor with a polyimide-coated photonics crystal fiber based on Brillouin dynamic grating," Journal of Lightwave Technology, 2020, 99: 5219-5224.

[77] Y. Dong, X. Bao, and L. Chen, "Distributed temperature sensing based on birefringence effect on transient Brillouin grating in a polarizationmaintaining photonic crystal fiber," Optical Letters, 2009, 34(17): 2590-2592.

[78] Y. Dong, L. Chen, and X. Bao, "High-spatialresolution time domain simultaneous strain and temperature sensor using Brillouin scattering and birefringence in a polarization- maintaining fiber," IEEE Photonics Technology Letters, 2010, 22(18): 1364-1366.

[79] K. Y. Song, S. Chin, N. Primerov, and L. Thevenaz, "Time-domain distributed fiber sensor with $1 \mathrm{~cm}$ spatial resolution based on Brillouin dynamic grating," Journal of Lightwave Technology, 2010, 28(14): 2062-2067.

[80] S. Chin, N. Primerov, and L. Thevenaz, "Subcentimeter spatial resolution in distributed fiber sensing based on dynamic Brillouin grating in optical fibers," IEEE Sensors Journal, 2012, 12(1): 189-194.
[81] W. Zou, Z. He, and K. Hotate, "Demonstration of Brillouin distributed discrimination of strain and temperature using a polarization-maintaining optical fiber," IEEE Photonics Technology Letters, 2010, 22(8): 526-528.

[82] A. Bergman, L. Yaron, T. Langer, and M. Tur, "Dynamic and distributed slope-assisted fiber strain sensing based on optical time-domain analysis of Brillouin dynamic gratings," Journal of Lightwave Technology, 2015, 33(12): 2611-2616.

[83] M. Pang, X. Jiang, W. He, G. K. L. Wong, G. Onishchukov, N. Y. Joly, et al., "Stable subpicosecond soliton fiber laser passively mode-locked by gigahertz acoustic resonance in photonic crystal fiber core," Optica, 2015, 2: 339-342.

[84] N. T. Otterstrom, R. O. Behunin, E. A. Kittlaus, Z. Wang, and P. T. Rakich, "A silicon Brillouin laser," Science, 2018, 360(6393): 1113-1116.

[85] E. A. Kittlaus, N. T. Otterstrom, P. Kharel, S. Gertler, and P. T. Rakich, "Non-reciprocal interband Brillouin modulation," Nature Photonics, 2018, 12(10): 613-619.

[86] A. Butsch, J. R. Koehler, R. E. Noskov, and P. S. J. Russell, "CW-pumped single-pass frequency comb generation by resonant opto-mechanical nonlinearity in dual-nanoweb fiber," Optica, 2014, 1: 158.

[87] K. Shiraki and M. Ohashi, "Sound velocity measurement based on guided acoustic-wave Brillouin scattering," IEEE Photonics Technology Letters, 1992, 4: 1177-1180.

[88] Y. Tanaka and K. Ogusu, "Temperature coefficient of sideband frequencies produced by depolarized guided acoustic-wave Brillouin scattering," IEEE Photonics Technology Letters, 1998, 10: 1769-1771.

[89] Y. Tanaka and K. Ogusu, "Tensile-strain coefficient of resonance frequency of depolarized guided acoustic-wave Brillouin scattering," IEEE Photonics Technology Letters, 1999, 11(7): 865-867.

[90] Y. Antman, A. Clain, Y. London, and A. Zadok, "Opto-mechanical sensing of liquids outside standard fibers using forward stimulated Brillouin scattering," Optica, 2016, 3(5): 510-516.

[91] D. M. Chow, M. A. Soto, and L. Thévenaz, "Frequency-domain technique to measure the inertial response of forward stimulated Brillouin scattering for acoustic impedance sensing," in 2017 25th Optical Fiber Sensors Conference (OFS), South Korea, 2017, pp. 10323.

[92] G. Bashan, H. H. Diamandi, Y. London, E. Preter, and A. Zadok, "Opto-mechanical time-domain reflectometry," Nature Communications, 2018, 9(1): 2991.

[93] D. M. Chow, Z. Yang, M. A. Soto, and L. Thévenaz, "Distributed forward Brillouin sensor based on local light phase recovery," Nature Communications, 
2018, 9(1): 2990.

[94] C. Pang, Z. Hua, D. Zhou, H. Zhang, L. Chen, $\mathrm{X}$. Bao, et al., "Opto-mechanical time-domain analysis based on coherent forward stimulated Brillouin scattering probing," Optica, 2020, 7(2): 176-184.

[95] B. Wang, Y. Dong, D. Ba, and X. Bao, "High spatial resolution: an integrative review of its developments on the Brillouin optical time-and correlation-domain analysis," Measurement Science and Technology, 2020, 31(5): 052001.

[96] A. W. Brown, B. G. Colpitts, and K. Brown, "Dark-pulse Brillouin optical time-domain sensor with $20 \mathrm{~mm}$ spatial resolution," Journal of Lightwave Technology, 2007, 25(1): 381-386.

[97] K. Kishida and C. H. Li, "Pulse pre-pump-BOTDA technology for new generation of distributed strain measuring system," Structural Health Monitoring and Intelligent Infrastructure, 2005, 1: 471-477.

[98] J. C. Beugnot, M. Tur, S. F. Mafang, and L. Tevenaz, "Distributed Brillouin sensing with sub-meter spatial resolution: modeling and processing," Optics Express, 2011, 19(8): 7381-7397.

[99] K. Hotate and T. Hasegawa, "Measurement of Brillouin gain spectrum distribution along an optical fiber with a high spatial resolution using a novel correlation-based technique: demonstration of $45 \mathrm{~cm}$ spatial resolution," in 13th International Conference on Optical Fiber Sensors, Kyongju, 1999, pp. 337-340.

[100] Y. Dong, D. Ba, T. Jiang, D. Zhou, H. Zhang, C. Zhu, et al., "High-spatial-resolution fast BOTDA for dynamic strain measurement based on differential double-pulse and second-order sideband of modulation," IEEE Photonics Journal, 2013, 5(3): 2600407. 